\title{
The regenerative response of cardiac interstitial cells
}

7 Laura Rolland ${ }^{1 \dagger}$, Alenca Harrington ${ }^{1 \dagger}$, Adèle Faucherre ${ }^{1}$, Girisaran Gangatharan ${ }^{1}$, Laurent

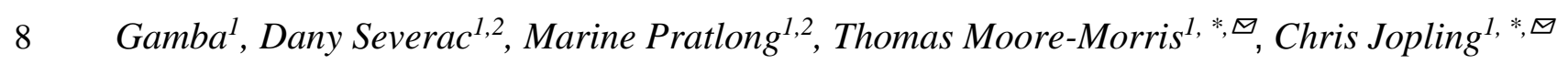

9

$10{ }^{1}$ Institute of Functional Genomics, University of Montpellier, CNRS, INSERM, LabEx ICST,

11

14

15

16

17

18

19

20
Montpellier, France

${ }^{2}$ Montpellier GenomiX, France Génomique, Montpellier, France

† These authors contributed equally: Laura Rolland, Alenca Harrington.

* These authors jointly supervised this work: Thomas Moore-Morris, Chris Jopling.

Email: thomas.moore-morris@inserm.fr; chris.jopling@igf.cnrs.fr 


\section{Abstract}

Understanding how certain animals are capable of regenerating their hearts will provide much

23 needed insights into how this process can be induced in humans in order to reverse the damage caused by

24 myocardial infarction. Currently, it is becoming increasingly evident that cardiac interstitial cells play

25 crucial roles during cardiac regeneration. To understand how interstitial cells behave during cardiac

26 regeneration, we performed single-cell RNA sequencing (scRNA-seq) of regenerating zebrafish hearts.

27 Using a combination of immunohistochemistry, chemical inhibition and novel transgenic animals, we were

28 able to investigate the role of cell type specific responses during cardiac regeneration. This approach

29 allowed us to identify a number of important regenerative mechanisms within the interstitial cell

30 populations. Here, we provide here a detailed insight into how interstitial cells behave during cardiac

31 regeneration and identify a number of novel features of these cells which will serve to increase our

32 understanding of how this process could eventually be induced in humans. 


\section{Introduction}

42 The very limited regenerative potential of the adult mammalian heart underlies an increasing prevalence of

43 heart failure(1). Studies using animal models such as the zebrafish and neonatal mice have shown that,

44 following a substantial loss of myocardium, regeneration can be achieved through cardiomyocyte

45 proliferation $(2,3)$. Furthermore, it has become increasingly evident that this process cannot occur without

46 a suitable environment that is provided by multiple interstitial cell populations. Notably, following injury,

47 clearing of cellular debris, neovascularisation and extracellular matrix scaffold constitution require the

48 highly coordinated activity of interstitial cells such as immune cells, endothelial cells and fibroblasts $(4,5)$.

Recent studies have harnessed the power of single-cell level analysis to overcome difficulties associated with heterogeneous cell populations such as cardiac fibroblasts and macrophages. This has

51 provided a more detailed overview of interstitial cell function after cardiac injury in adult mice and during

52 cardiac regeneration in neonates $(6,7)$. Although the regenerating neonatal mouse heart is highly relevant

53 for identifying mechanisms that could help promote adult human heart regeneration, it is also actively

54 remodeling when subjected to injury, meaning certain features relevant to achieving regeneration in the

55 adult heart may be missing. The zebrafish represents a complementary model for exploring cardiac

56 regeneration as quiescent adult zebrafish myocardium is able to regenerate following significant injury(2,

$578)$

To further understand the process of cardiac regeneration in adult zebrafish, we have performed

59 single cell analysis of interstitial cell populations in regenerating hearts. Furthermore, we provide a rigorous

60 quantification of the different cell types present in the zebrafish ventricle, including cardiomyocytes,

61 endothelium, epicardium, fibroblasts, macrophages and erythrocytes. Our analysis has revealed intriguing

62 properties of fibroblasts, endothelial cells and macrophages that support cardiac regeneration in adult 63 zebrafish. 
Fibroblasts are involved in multiple processes associated with the cardiac response to injury and

65

66

67

68 have previously been shown to play a crucial role during cardiac regeneration in zebrafish(9-11). Our data indicates many similarities in the injury response between zebrafish cardiac fibroblasts and their adult mammalian counterparts, however, we have also identified significant differences, most notably a disparity in myofibroblast gene expression. Endothelial cells make up the bulk of the cardiac interstitial population. Recent studies have determined that endothelial neovascularisation of the wound area is key for cardiac regeneration. This process lays down a framework over which the regenerating myocardium can be formed(5). Here we have determined that tall, a gene essential for endocardial development, is required for cardiac regeneration in adult zebrafish. The macrophage response during cardiac regeneration also plays a pivotal role in ensuring a successful outcome(12-15). For a long time, the balance between inflammation and regeneration has been regarded as one of the key elements of this process. Interestingly, our data indicates that the resident macrophages present in the uninjured adult zebrafish heart appear to display an inflammatory M1 signature, which, following injury, is rapidly attenuated in conjunction with the arrival of recruited M2 macrophages. Furthermore, we have also determined that the matrix metalloproteinase, mmp14, is primarily expressed by recruited M2-like macrophages and plays a crucial role in allowing them to invade the damaged tissue.

Our study underlines the importance and variety of interstitial cell functions that support adult zebrafish heart regeneration. Furthermore, we have also compared our findings with published single cell RNA sequencing (scRNA-seq) studies of the interstitial cellular response in regenerating neonatal mice and non-regenerating adult mice following myocardial infarction(6, 7). In so doing we have endeavored to provide a balanced overview of the similarities and differences between regenerating and non-regenerating models. 


\section{Results}

Single cell sequencing of regenerating zebrafish ventricle.

In order to analyse the regenerative response of different interstitial cell populations, we adopted a scRNAseq strategy. Following optimization of dissociation conditions and FACS-sorting of viable nucleated cells, we performed scRNA-seq (10x Chromium) of uninjured, sham operated and amputated (3 days, 7 days and 14 days post-amputation (dpa)) adult zebrafish ventricles. Altogether, after quality control, we obtained 18,739 transcriptional profiles and found that samples from uninjured and sham-operated zebrafish were largely comparable (Fig.1.A and Suppl Fig.1.A, Suppl Table I). Unbiased clustering of cells from uninjured and amputated hearts revealed 14 clusters comprising 9 distinct cell types (Fig.1.B, Suppl Fig 1.B and Suppl data.1 and 2). Major cell types included $c d h 5^{+}$endothelium, mpeg $1.1^{+}$myeloid cells (macrophages), $t c f 21^{+}$ epicardium/epicardium-derived cells (EPDCs), baal $^{+}$erythrocytes and sla2 ${ }^{+}$lymphoid cells (Fig.1.B, C). included $\mathrm{mpx}^{+}$neutrophils, itga $2 \mathrm{~b}^{+}$thrombocytes and $\operatorname{sox} 10^{+}$cardiac neural crest (and $r$ spol ${ }^{+}$neural crest derivatives) (Fig.1.B, C). Although we could detect a small population of $m y l 7^{+}$cardiomyocytes, these cells were largely absent from our data set presumably because their size was not compatible with our scRNA-

103 seq pipeline (Fig.1.B, C). Very rare "hybrids" i.e. expressing markers of multiple lineages, formed 104 protrusions from cell-type specific clusters aimed in the direction of another cell type, as most obviously observed with the myocyte cluster (Fig.1.B). Cell types were clearly separated in the UMAP plots, with an exception of the myeloid and lymphoid lineages. These populations included converging sub-populations

107 characterized by a high expression of cell-cycle related genes (e.g. $c d k 1$, mik67) (Fig.1.C-E and Suppl data.1 108 and 2). 
112 responses to injury. Previous studies have established that the adult zebrafish heart is mainly composed of

113 cardiomyocytes and endothelium(16), but the proportions of other key cell types, including resident

114 macrophages and epicardium/EPDCs has not previously been described. Using IHC and several reporter

115 lines, we were able to assign a cell-type identity to $91.8 \%$ of DAPI ${ }^{+}$nuclei in the ventricle (Fig.1.F-L).

116 These included Mef2 $\mathrm{c}^{+}$cardiomyocytes (32.8\%), Tg(flila:GFP)yl ${ }^{+}$endothelial cells (46.2\%),

$117 \mathrm{Tg}(\text { gatal:dsred })^{+}$erythrocytes $(8.6 \%), \mathrm{Tg}(\text { mpeg 1.1:mCherry })^{+}$macrophages $(2.4 \%), \mathrm{Tg}\left(\mathrm{colla2}: \mathrm{mCherry}^{+}\right.$

118 epicardium/EPDCs (1\%) and a-smooth muscle actin (Acta2) ${ }^{+}$smooth muscle cells (0.8\%) (Fig.1.G-L). It

119 was essential to clearly identify and quantify zebrafish erythrocytes as they are nucleated in this species.

120 We were unable to assign an identity to $8.2 \%$ of nuclei that may include cells with a weak reporter/IHC

121 signal and rare populations such as lymphocytes or CNC-derived cells. Hence, as in the adult mouse heart,

122 the endothelial and cardiomyocyte populations represented the most abundant cell types(17). However, the

123 uninjured adult zebrafish heart presented a relatively low number of epicardium and epicardial-derived cells

124 (EPDCs) such as fibroblasts. Indeed, we evaluated that epicardium/EPDCs represented $1 \%$ of the cells in adult the zebrafish heart. In comparison $15 \%$ of the adult mouse heart is comprised of fibroblasts(17).

\section{Macrophages}

127 Our analysis revealed 3 macrophage clusters (MC1-3), including MC1 that was predominant in uninjured

128 hearts. The top gene associated with these resident MC1 macrophages versus other myeloid clusters was

$129 \quad c x c r 3.3$, a ligand scavenging receptor associated with reduced macrophage mobility(18). These cells also

130 expressed the highest levels of markers of activated M1 macrophages ( $t n f a, i l 1 b, c d 40$, il6r) and neutrophil-

131 recruiting chemokines (cxcl8a, csf3b) (Suppl Fig.1.D and Suppl data.1). MC2 was predominant at 3dpa,

132 and expressed high levels of genes associated with M2-like properties, including ctsc and $c$ lqa (Suppl

133 Fig.1.D and Suppl data.1). Also, MC2 macrophages expressed genes associated with recruited macrophages

134 such as ccr2(19) and apoeb(20) as well as tissue healing including the copper chaperone atoxl(21) (Suppl

135 Fig.1.D and suppl data.1). MC3 was characterized by a very strong cell-cycle related gene signature,

136 including mki37, top2a, pcna and $c d k 1$ (Fig.1.D, D1, D2 and Suppl data.1). In support of this, we were able 
137 to directly observe proliferating EdU-labelled macrophages in regenerating ventricles by 138 immunohistochemistry (IHC) analysis (Fig.1.E).

\section{Endothelium}

Based on cell counts, we determined that $46.2 \%$ of cells in the ventricle were endothelial/endocardial. Interestingly, our scRNA-seq data clearly showed that, both at baseline and following resection, endocardial endothelium had both an endothelial (cdh5) and mesenchymal (pdgfra, colla2) signature, as previously observed in mouse(22). We obtained four endothelial cells clusters, all showing strong expression of pan-endothelial markers aqp8a.1, $c d h 5$ and $v w f$ (Suppl Fig.1.B and Suppl data.2). Endothelial clusters (EC) 1 and 2 were abundant in control hearts. EC1 was characterized by expression of relatively high levels of collagen (collala/b, colla2) (Suppl Fig.1.E and Suppl data.1). EC2 was characterized notably by $n p p c$, a key regulator of vascular homeostasis(23) whose receptor $n p r 3$ was expressed by EPDCs at baseline (Suppl Fig.1.E and Suppl data.1). EC3 and EC4 increased in size over time following amputation (Fig.1.B1-B4). EC3 expressed relatively high levels of several heme-binding genes (hbba1, hbba2, hbaa1, hbaa2), albeit at a far lower level than erythrocytes (Fig.1.C and Suppl Fig.1.E and Suppl data.1 and 2.). Markers of venous endothelium, such as $k d r l$, were not specific to any EC cluster (Suppl Fig.1.E). Markers of lymphatic endothelium proxla and lyvelb were expressed by a small subset of endothelial cells that did not segregate to any specific cluster (Suppl Fig.1.E and Suppl data.1).

\section{Epicardium/EPDCs}

Within our dataset we could clearly delineate two Epicardial/EPDC clusters. Epicardium/EPDC cluster 1 (FB1), was characterized by a high expression of genes associated with extracellular matrix organization (adamstl2, coll8alb) and integrin binding (edil3a, hapln1a) (Suppl Fig.1.F and Suppl data.1). Cells in FB2 were notably characterized by a high expression of genes involved in complement activation $(c 4, c 4 b, c \sigma)$ (Suppl Fig.1.F and Suppl data.1). Proportionately, FB1 was most abundant in unamputated hearts, whereas cell numbers in FB2 increased following injury (Fig.1.B1-B4). We were able to clearly identify epicardium 
161

162

163

164

165

166

167

168

169

170

171

172

173

174

175

176

177

178

179

180

181

182

183

184

and epicardial-derived fibroblasts based on their expression of the previously described fibroblast specific

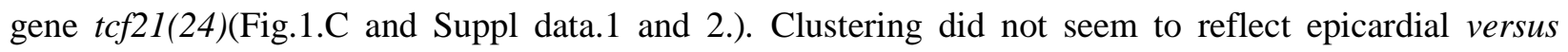
epicardium derived cells. For example, aldhla2 ${ }^{+}$and $\mathrm{clu}^{+}$epicardial cells were present in subsets of both FB1 and FB2 (Suppl Fig.1.F and Suppl data.1).

\section{Neural crest}

We were also able to identify a sox $10^{+}$population of cardiac neural crest (CNC)-derived cells which were assigned to the FB2 cluster, probably because of relatively low numbers of cells (Suppl Fig.1.B). We classified these cells as $\mathrm{CNC}$ derived based on the high expression of markers of neural crest cells ( $\operatorname{sox} 10^{+}$, pax3 $a^{+}$, apoda. $\left.1^{+}\right)$and CNC-derived mesenchyme $\left(r s p o 1^{+}\right.$, gyg $1 b^{+}, \operatorname{csrp} 2^{+}$, hand2 $2^{\text {high }}$ ) (Fig.2.A and Suppl data.1 and 2.). Strikingly, these cells did not express the epicardium/EPDC-specific genes, $t c f 21$ and $t b x 18$ (Fig.2.A) nor the FB2-specific gene, adh8a (Suppl data.1). Within the CNC cluster, we could clearly distinguish sox $10^{\text {high }}$;rspol ${ }^{-}$and $\operatorname{sox} 10^{\text {low }}$; rspol $^{+}$sub-clusters. Previous studies in zebrafish have shown that sox10-expressing cells are concentrated in the atrioventricular valves(25). In mice, Rspol has recently been reported to be expressed in epicardial cells(7). IHC analysis of neonatal mouse hearts confirmed that RSPO1 was indeed associated with the epicardial layer (Suppl. Fig 2.A). Similarly, IHC revealed that rspo $^{+}$cells were also most abundant in the zebrafish ventricle epicardial layer (Fig.2.B). However, reanalysis of the scRNA-seq data by Wang et al.(7) confirmed that, in contrast to what we observed in zebrafish, murine Rspol $^{+}$cells were clearly expressing epicardial markers such as Tcf21 and Tbx18 (Suppl Fig 2.B), underlining a divergence in the profiles of Rspo $^{+}$cells in these species.

\section{A hallmark of activated mammalian cardiac fibroblast is absent in zebrafish.}

Following injury, we observed a strong fibrotic response, which was initiated in epicardium/EPDCs, notably with a robust upregulation of periostin (postnb) and fibronectin (fnla/b) expression(26) (Suppl Fig.2.C and Suppl data.1 and 2). In contrast, acta2, a gene associated with injury-induced myofibroblast activation in mammals(26), was not upregulated at any time point in the epicardium/EPDC lineage (Suppl 
Fig.2.C). To confirm this observation we performed cardiac amputations on $\operatorname{Tg}($ colla2:mCherry) zebrafish followed by IHC for Acta2 at 3dpa and 7dpa (Fig.2.C-J). Surprisingly, although the number of Col1A2:mCherry ${ }^{+}$cells increased following injury, this was not accompanied by an increase in Acta2 expressing cells (Fig.2.D,I,J). Elevated Acta2 production can be observed in non-regenerating adult mammalian hearts following injury and, similarly, an increase in Acta2 production has also been observed in adult zebrafish mutants which are unable to regenerate their hearts(27). Based on these observations we surmised that inhibiting cardiac regeneration in adult zebrafish may also lead to an increase in Acta2

192 production and allow us to further investigate whether or not this was associated with fibroblasts. The pan-

193 MMP inhibitor GM6001 has previously been reported to significantly inhibit zebrafish cardiac regeneration(28). In agreement with this we also found that treating adult zebrafish with GM6001 following apical resection resulted in a failure to regenerate at 30dpa and the formation of a large fibrin/collagen scar (Fig.2.H). Using this protocol we performed cardiac amputations on $\operatorname{Tg}$ (colla2:mCherry) zebrafish followed by IHC for Acta2 in the lower ventricle at 3dpa and 7dpa (Fig.2.E,G,I,J). Interestingly, inhibiting cardiac regeneration with GM6001 led to a significant increase in the number of interstitial Acta2 ${ }^{+}$cells at 7dpa (Fig.2.G,J). However, Acta2 did not co-localise with colla2:mCherry ${ }^{+}$, indicating that cell types other than fibroblasts were upregulating Acta2 expression (Fig.2.E,G). This is in agreement with a recent study that reported the expression of smooth muscle-specific genes outside of the epicardial lineage, notably in the endocardium(29). To determine whether the expression of ACTA2 by fibroblasts varies between species we re-analysed 2 previously published scRNA-seq datasets from non-regenerating adult mice and regenerating neonatal mice after myocardial infarction(6, 7)(Suppl Fig.2.C). Interestingly, the sustained expression of the fibrosis associated gene, Postn, 3 days after injury appeared remarkably similar between adult mice and zebrafish (Suppl Fig.2.C). On the other hand, following myocardial infarction in adult mice and P8 neonates there was a robust upregulation of Acta2 expression by fibroblasts 3 days post injury, a

208 feature which was absent in the adult zebrafish after cardiac injury (Suppl Fig.2.C). The picture in 209 regenerating P1 neonate fibroblasts was far less clear as they already expressed Acta2 at baseline along 210 with high levels of Postn, presumably because the P1 neonatal heart is still undergoing widespread 
211 remodeling (Suppl Fig.2.C). Taken together, our data indicates that collagen-producing cardiac fibroblasts

212 in adult zebrafish do not upregulate Acta2 in response to injury.

\section{Tal1 is a regulator of the endothelial regenerative response.}

214 The zebrafish endothelial response to injury involves a rapid change in gene expression followed by wound 215 neovascularization, a process which lays the foundation for the proliferating cardiomyocytes to regenerate

216 the missing myocardium(5). A number of genes have been directly linked to the endothelial regenerative 217 response in zebrafish such as vegfaa, aldh1a2, and notch1b(30-32). We could not detect any significant 218 upregulation of any of these genes, which could be due to rapid changes in their expression occurring 219 outside of the time points analysed here, as reported for vegfaa(30). On the other hand, although the average 220 level of expression of notchlb in the endothelium did not change significantly we could detect an increase $221(8 \%)$ in the proportion of endothelial cells expressing this gene at 3dpa compared with uninjured controls. 222 The re-expression of developmental genes is also a hallmark of regenerating endothelium(5). We found 223 that the proportion of cells expressing genes required for endothelial development increased during cardiac 224 regeneration (foxcla, foxclb, (Suppl Fig 3.A,B)(33)). The BHLH transcription factor Tal1 is also essential 225 for endocardial development and for maintaining endocardial identity, in particular Tal1 is required for 226 establishing endothelial Tjp1 tight junctions(33). Previous research has shown that tall is upregulated 227 during cardiac regeneration in zebrafish(34). In agreement with this, our scRNA-seq analysis indicates that 228 there was an increase in the proportion of tall expressing endothelial cells during regeneration (Fig.3.A,B).

229 This increase peaked at 7dpa before returning to pre-injury levels by $14 \mathrm{dpa}$ (Fig.3.B). Without detailed 230 lineage tracing we are presently unable to determine whether the increase in tall expressing endothelial 231 cells was due to the proliferation of existing tall expressing cells or because more endothelial cells have 232 begun producing tall de novo. To confirm our scRNA-seq data, we performed IHC for Tall on adult $233 T g(f l i l a: G F P) y 1$ zebrafish cardiac sections and were able to clearly observe Tal1 positive endothelial cells 234 (Fig.3.C-E). Because Tal1 is an obligate dimer and can form complexes with a variety of proteins which 235 will subsequently dictate which transcriptional programs to activate/deactivate(35), we extended our 
analysis to known co-factors of Tal1. LIM only 2 (LMO2), forms a multi-protein complex with TAL1 and directs it towards specific targets genes. Previous research indicates that in the absence of LMO2, TAL1 is able to target other genes for expression/repression(35). We found that $l m o 2$ was downregulated during the early stages of regeneration (Suppl Fig 3.C). In order to analyse this in more detail we reclustered tall $^{+}$ endothelial cells to identify changes in gene expression associated with this sub-population (Fig.3.F). Interestingly, this analysis revealed that although $l m o 2$ expression was evenly distributed in 3 of the tall positive $\left(\right.$ tall $\left.^{+}\right)$clusters (T1,T2,T3) it was reduced in the fourth (T4)(Fig.3.G). Furthermore, the expression of the $\mathrm{Lmo2}^{-} / \mathrm{Tall}^{+}$target gene cgnll(35), a component of tight junctions and implicated in

244 neovascularization, shows the highest level of expression in cluster T4 (Fig.3.H). We could also observe a 245 downregulation of $l m o 2$ at 3dpa and 7dpa specifically in tall $^{+}$endothelial cells (Fig.3.I). Conversely, cgnl1 246 became upregulated in tall $^{+}$cells at 7dpa (Fig.3.J). Although these observations were below the threshold 247 of significance $(\mathrm{P}<0.05)$, this evident trend prompted us to target tall directly to determine whether this core endocardial developmental gene could be involved in cardiac regeneration. In order to achieve this we generated a transgenic zebrafish line which can express a dominant negative Tal1 isoform specifically in endothelial cells following treatment with tamoxifen (fliEP:Ert2CreErt2; fliEP:loxRFPlox:DNtal) (Suppl Fig 4.A-G). The dominant negative Tal1 isoform lacks the basic DNA binding domain but is still able to form multi-protein transcription complexes and thus inhibit native Tal1 associated transcription(36). We first assessed whether this transgenic line was functional by inducing DN tal expression during early zebrafish development. This procedure caused cardiac developmental defects reminiscent of those previously described for tall knockout zebrafish (Suppl Fig 4. E-G). To determine whether tall is required for heart regeneration we induced expression of DN tal prior to cardiac resection. Histological staining of heart sections at 30dpa revealed that expression of DN tal inhibited the regenerative process, as evidenced by a large fibrin/collagen scar (n=5) (Fig.3.K-P.). Lastly, to determine whether the expression of tall in the endothelium is zebrafish specific we re-analysed 2 previously published scRNA-seq datasets from nonregenerating adult mice and regenerating neonatal mice after myocardial infarction and observed that Tall 
is present within the endothelial populations(6, 7) (Suppl Fig.4.H). Taken together these data indicate that tall is a key regulator of the endothelial response during cardiac regeneration.

\section{MMP14 expressing macrophages are required for cardiac regeneration.}

264 Macrophages play a crucial role during cardiac regeneration(15). We were able to identify 3 clusters of macrophages within our scRNA-seq data set. As described earlier, MC1 represented a resident population of inflammatory/M1 macrophages, which was predominant in uninjured hearts (Fig.4.A). MC2 was comprised of recruited macrophages, which were abundant at 3dpa, persisted through 7dpa and resolved by 14dpa (Fig.4.A). This cluster was enriched for transcripts commonly associated with M2 polarised macrophages such as $c d 9 a / b$, inflammasome associated genes (txnipa, caspa and atp13a2) and csf1ra(3740) (Fig.4.A and Suppl Fig.5 A-F and Suppl data.1). Furthermore, MC2 was also enriched for genes previously described to be associated with regenerating macrophages in adult zebrafish (fabplla, lgals $9 l 1$ and lgmn(41)) (Suppl Fig 5.G-I and Suppl data.1). Lastly, MC3 was enriched for genes associated with the cell cycle such as pcna and mki67 indicating that macrophage proliferation also occurs during cardiac regeneration in zebrafish, similar to the proliferation observed in mouse hearts following myocardial infarction (MI)(42) (Suppl Fig 6.A,B and Suppl data.1). 
following myocardial injury(19). During cardiac regeneration in P1 neonatal mice there was an increase in macrophage $C c r 2$ expression 1 day after injury, which is also associated with an increased expression of Tnfa and $i l 1 b$, indicating a wave of pro-inflammatory macrophages are recruited to the heart shortly after injury (Suppl Fig.8). Similarly, in adult mice after MI there is an influx of $C c r 2$ expressing macrophages at 3 days post injury which coincides with an increase in $I l l b$ expression (Suppl Fig.8). Our own data indicates that during cardiac regeneration in adult zebrafish $c c r 2$ was expressed at low levels 3 days after injury, predominantly in the recruited MC2 population (Suppl Fig.8). Although it appears from our data that resident macrophages (MC1) expressed higher levels of tnfa and $i l 1 b$ than the recruited population (MC2)

294 (Suppl Fig.8), we cannot rule out the possibility of a rapid influx of pro-inflammatory macrophages at an 295 earlier timepoint, as observed in neonatal mice. In support of this notion, the expression profiles for tnfa and $i l l b$ at 3 days post injury followed a similar pattern in both neonatal mice and adult zebrafish. In particular there was a marked reduction in expression of both these genes at 3 days post injury compared to uninjured controls (Suppl Fig.8). Further analysis will be required at earlier timepoints to fully decipher the macrophage inflammatory response during cardiac regeneration in zebrafish. macrophages to CXCL chemokine signals. In mammals, G protein coupled chemokine receptor 3 (CXCR3) signalling is required for recruiting macrophages to the site of injury/infection(46). Previous reports indicate that zebrafish possess 2 orthologs of this gene, cxr3.2 and cxcr3.3. Although cxcr3.2 appears to be a

304 functionally active receptor, $\operatorname{cxc} 3.3$ lacks the ability to activate downstream signaling pathways and hence 305 acts as a scavenger of $\operatorname{cxcr} 3$ ligands, effectively dampening down the $\operatorname{cxcr3.2}$ response(18). Although all

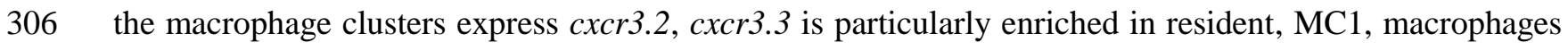
307 which would presumably reduce their ability to respond to cxcr3 ligands (Suppl Fig.9.A,B and Suppl 308 data.1). Matrix metalloproteinases (MMP) are also well established players in wound healing and 309 regeneration and have been linked to a variety of processes that are necessary to resolve damaged tissue ${ }^{10}$. 310 Transcriptomic analysis of zebrafish heart regeneration has consistently identified an upregulated 
311 expression of various MMPs during this process(28, 34, 47). Furthermore, MMPs have been shown to be

312 involved in zebrafish fin regeneration(48), newt limb regeneration(49) and salamander limb

313 regeneration(50). We initially assessed MMP expression during cardiac regeneration by qPCR and

314 observed a dynamic response during this process, in particular the expression of $m m p 2$ and $m m p 14 a / b$

315 (zebrafish possess 2 MMP14 orthologs) increased substantially (Fig.4.B). Analysis of our scRNA-seq data

316 indicates that $m m p 14 a / b$ were predominantly expressed by fibroblasts and macrophages. In particular

$317 m m p 14 a$ was restricted to the fibroblast population and was absent in macrophages while, $m m p 14 b$ appears

318 to be expressed by both populations (Fig.4.C and Suppl data.1). Of particular interest, we also observed

319 that mmp14b is predominantly expressed by the recruited MC2 macrophages (Fig.4.D and Suppl data.1).

320 To confirm that macrophages express MMP14 during regeneration we performed IHC for MMP14 on

321 regenerating zebrafish and P1 neonatal mouse hearts (7dpa). In this manner, we were able to clearly detect

322 MMP14 positive macrophages present during regeneration in both species (Fig.4.E-L). To gain further

323 insight into MMP14 expression by macrophages we re-analysed 2 previously published scRNA-seq

324 datasets from non-regenerating adult mice and regenerating neonatal mice after myocardial infarction(6,

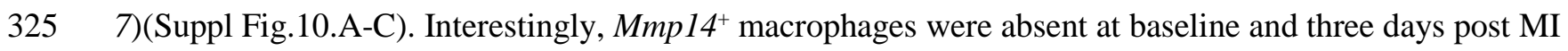

326 in both adult and neonatal mice (Suppl Fig.10. B, C). This is in contrast with zebrafish, in which mmp14b

327 expressing macrophages were present in uninjured hearts and peak at 3dpa (Supp Fig.10.A). To determine

328 whether Mmp14 is required for cardiac regeneration we employed a specific inhibitor of this protein,

329 NSC40520, which blocks the collagenolytic activity of MMP14 but not its ability to activate other targets

330 such as MMP2(51). Histological analysis of cardiac sections taken from 30dpa adult zebrafish indicated

331 that NSC40520 treatment significantly impaired cardiac regeneration, resulting in the formation of a

332 fibrin/collagen scar (n=5) (Fig.5.A-D). To determine whether Mmp14 inhibition disrupts the macrophage

333 regenerative response, we repeated these experiments at $3 \mathrm{dpa}$, a time point when $\mathrm{M} 2 / \mathrm{Mmp} 14^{+}$recruited

334 macrophages were most abundant. IHC analysis of cardiac sections revealed substantial numbers of

335 macrophages in the wound region at 3dpa in untreated control samples (n=5) (Fig.5.E-G). Strikingly,

336 macrophages were noticeably absent in the wound region of NSC40520 treated samples (n=5) (Fig.5.H-J). 
Based on these observations, we performed scRNA-seq analysis to assess what effect Mmp14 inhibition had on the macrophage population. In order to identify the early events affected by NSC40520 treatment we focused on 3dpa when recruited mmpl4b expressing macrophages are most abundant. Analysis of the fibroblast population within this dataset indicates that overall there are no dramatic changes in the expression of genes associated with the fibrotic response following inhibitor treatment (Suppl Fig.11.A-D).

342 However, the expression of mmp14a and mmp14b did increase, which may reflect an attempt by these cells

343 to counteract the Mmp14 inhibition (Suppl Fig.11.E, F). Within the macrophage population we could 344 identify 2 clusters of recruited macrophages, MC2a and MC2b which were enriched for $m m p 14 b$ and $c d 9 b$

345 (Fig.5. K, L and Suppl Fig.12.A, B). Furthermore, we were able to identify a number of genes whose expression was significantly upregulated in $m m p 14 b$ enriched macrophages following inhibitor treatment.

347 Notably we observed a robust upregulation of fabplla and lgals9l expression, both of which have previously been associated with regenerating macrophages(41)(Suppl Figs.12.C, D). Of potential interest the heparin-binding epidermal growth factor-like growth factor, hbegfb, which is cleaved and activated by MMP14(52), is specifically upregulated in recruited macrophages following inhibitor treatment (Fig.5.M collagenolytic activity of Mmp14 results in defective migration of M2 recruited macrophages into the wound region.

\section{Discussion}


a notable difference in the proportion of cells derived from the epicardial lineage. In particular, the number of fibroblasts present in the adult zebrafish heart (1\%) was considerably lower than what has been reported for the adult mouse heart $(15 \%)(16)$. In mammals, loss of cardiac tissue due to myocardial infarction (MI) triggers the differentiation of fibroblasts into myofibroblasts which are involved in replacing the lost tissue with a fibrotic scar(26). Although this initial phase is essential for maintaining cardiac integrity, the fibrotic response can spread throughout the heart leading to impaired cardiac function and ultimately failure. Indeed, it has been proposed for some time that the difference between scarring and regeneration could be influenced by the fibrotic response to injury(53). One feature of this response involves $\alpha$ Smooth Muscle Actin (ACTA2), a gene commonly restricted to smooth muscle cells, becoming specifically upregulated in myofibroblasts(26). Our data, combined with re-analysis of scRNA-seq data from mouse models, showed that, following injury, the epicardial/fibroblast lineage strongly upregulated a number of myofibroblastassociated genes such as Postn/postnb and Fnl/fnla in both species. However, this was not the case for Acta2, which was upregulated in non-regenerating P8 and adult mouse, relatively weakly upregulated in regenerating P1 neonatal mouse, and low/absent in zebrafish. Interestingly, in agreement with a very recent study (54), we found that the presence Acta2 ${ }^{+}$interstitial cells was significantly increased when regeneration was blocked in zebrafish hearts. Although these cells were not labelled by the colla2:mCherry reporter, Allanki et al have revealed that, at least in the cryoinjury model, a proportion of them were of epicardial origin. A relative lack of Acta2 upregulation in zebrafish interstitial cells during regeneration could indicate a reduction in myofibroblast differentiation, or the nature of this transition, and potentially the associated fibrotic response. Another possibility involves the ability of ACTA2 to enable myofibroblasts to contract during the process of wound healing(55). Indeed, wound contraction following injury serves to decrease the amount of tissue which needs to be repaired. In the heart this would allow the damaged tissue to be repaired rapidly in order to avoid rupture, a process which most likely supersedes regeneration in adult mammals. It is therefore conceivable that in regenerating tissue, wound contraction may not be required and could even inhibit regeneration by impeding certain regenerative processes, such as neovascularization, which may benefit from an open/relaxed wound environment. Further research will be required in order to 
determine whether the lack of Acta2 observed in zebrafish fibroblasts can be linked to reduced myofibroblast differentiation and fibrosis, or whether this affects a specific feature of myofibroblasts, such as contractility.

Our data also highlights that the choice of genetic marker used to isolate and study fibroblasts is critical. Indeed, we found that genes that have previously been reported to be upregulated in fibroblasts in 393 zebrafish, such as rspol(9) were in fact expressed by $\operatorname{tcf} 21^{-}$neural crest derivatives. The origin of $r s p o 1^{+} ; t c f 21^{-}$cells in the epicardial layer and whether they play a role in cardiac regeneration will require further investigation.

Following cardiac injury in adult zebrafish there is a rapid endothelial response resulting in wound neovascularization. This precedes the expansion of proliferating cardiomyocytes which will repopulate and ultimately regenerate the missing tissue. Our data indicates that tal1 plays an essential role in the endothelial regenerative response and that Tal1 inhibition, for example by the expression of a dominant negative isoform as we have shown here, impedes cardiac regeneration. Tal1 has been linked to a number of endothelial processes which may affect the regenerative response, such as the regulation of endocardial 402 cell-cell contacts, endocardial identity and neovascularization(56, 57). Although tall expression does not 403 increase dramatically during cardiac regeneration, based on previous research it is reasonable to speculate 404 that the genetic programs that Tal1 regulates are, very likely, dictated by its co-factors(35). Indeed, a 405 combination of chromatin immunoprecipitation (ChIP) and transcriptomic analysis has shown that, in the absence of Lmo2, TAL1 relocates to different DNA target sites where it regulates alternative genetic programs. Our scRNA-seq data indicates that the expression of the Tal1 co-factor, Imo2, decreases in $\operatorname{tall}^{+}$ endothelial cells at 7dpa. Intriguingly, we also observed an increase in expression of the tight junction associated gene cgnll at 7dpa in tall $^{+}$endothelial cells. Cgnll has also been implicated in neovascularization(58). Because Tal1 has previously been described to regulate endothelial tight junctions

411 during endocardial development it is tempting to speculate that this process has been disrupted following 412 Tal1 inhibition, resulting in defective cardiac regeneration. 
414 regeneration has been demonstrated in a variety of different species(12, 15, 59). Our scRNA-seq data has

415 revealed a number of interesting features associated with macrophages during cardiac regeneration. Firstly,

416 although resident macrophages in the uninjured heart appear to have a more pro-inflammatory signature

417 compared to the recruited 3dpa population we observed, we cannot rule out the possibility that the situation

418 may be reversed at an earlier timepoint, as appears to be the case in regenerating neonatal mouse hearts.

419 Previous reports have indicated that during regeneration in neonatal mice there is no recruitment of pro-

420 inflammatory $C c r 2$ expressing macrophages $(60)$. However, analysis of published scRNA-seq data indicates

421 a rapid influx of $C c r 2$ expressing macrophages 1 day post MI coincident with elevated $T n f a$ and $I l 1 b$

422 expression, an event which may have been missed previously. Furthermore, at 3 days post MI in

423 regenerating neonatal mouse hearts, despite being more numerous, macrophages expressed lower levels of

$424 T$ Tnfa and $I l 1 b$ compared to sham operated animals. We observed a similar increase in macrophage cell number but reduction in tnfa and $i l l b$ expression within these cells in zebrafish hearts 3 days after injury.

These data suggest that the inflammatory response of macrophages in regenerating adult zebrafish hearts is response(42). More focused studies will be required in order to determine whether the initial macrophage response, occurring during the first hours/days after injury, reflects any difference in regenerative capacity between adult zebrafish and mammals.

Our data also indicates that zebrafish possess a potentially unique mechanism for regulating the 435 macrophage response to CXCL chemokine signals. CXCR3 and its ligands are responsible for recruiting macrophages to sites of injury/infection(61). Previous research indicates that zebrafish possess 2 CXCR3 

zebrafish, all macrophages express $\operatorname{cxcr} 3.2$ in relatively equal proportions, however, resident macrophages also express nearly 2 fold more $\operatorname{cxcr} 3.3$ than recruited macrophages and as such it is fair to assume that this

442 will reduce their ability to respond to Cxcr3 ligands. This may provide zebrafish with an elegant mechanism

443 for fine-tuning the resident M1 macrophage response to Cxcl ligands. For example, modulating Cxcr3 444 signalling may play a role in blunting the inflammatory response of the M1 resident macrophages during 445 cardiac regeneration. It could also potentially serve to reduce the mobility of resident macrophages and help 446 to maintain this population within the heart. collagenolytic activity of Mmp14 resulted in defective migration of macrophages into the injury site and a subsequent failure to regenerate the myocardium, leading to the formation of large collagen/fibrin scar. Our scRNA-seq analysis of Mmp14 inhibited macrophages indicated that even at this early time point the expression of a number of genes associated with the regenerative response have become misregulated. Of

454 potential interest is HB EGF which has been linked to a variety of processes important for cardiac 455 regeneration $(63,64)$. However, expression of $h b e g f b$ appears to increase when cardiac regeneration fails 456 following Mmp14 inhibition. In this context HB EGF has previously been shown to have detrimental effects 457 on the remodeling process after myocardial infarction in mammals by enhancing fibroblast activation and 458 invasion(65). It could therefore play a role in the development of the substantial fibrin/collagen scar that is 459 present at 30dpa when Mmp14 is inhibited. Re-analysis of scRNA-seq data from adult mice and neonates 460 following myocardial infarction showed that, conversely to what we observed in zebrafish, Mmp14 461 expressing were not present in the myocardium prior to injury. Whether the presence of Mmp14b-producing resident macrophages confers zebrafish with some kind of regenerative advantage, perhaps by allowing 
resident macrophages to respond in a similar manner to recruited macrophages, will require further investigation. However, in stark contrast to our own observations in zebrafish, it appears that in adult mice, MMP14 plays a deleterious role after cardiac injury(62). Indeed MMP14 heterozygote KO mice display a marked improvement in survival post MI due to reductions in infarct size, left ventricular dilation and compensatory hypertrophy. Furthermore, there is also a significant reduction in the number of macrophages localised to the infarct area in MMP14 +/- mice, similar to our own observations in regenerating zebrafish hearts when Mmp14 is inhibited. More recently, it has been shown that macrophage specific deletion of MMP14 in adult mice reduces left ventricular dysfunction following MI(66). It appears that loss of MMP14 in macrophages results in attenuated, Tgf $\beta$ dependent, fibrosis. Our own data indicated that mmpl4b expressing macrophages are recruited to the wound site a few days after injury, similar to observations in mouse MI models. Targeting Mmp14 in zebrafish with a specific inhibitor disrupted cardiac regeneration, which appears to be the complete inverse of the situation described in adult mice. Why this is the case remains unclear. It is possible that zebrafish Mmp14b possesses properties that are absent from mammalian MMP14, or this could be due to the expression of $m m p 14 b$ by resident macrophages in uninjured zebrafish hearts, a feature that is not present in neonatal or adult mouse hearts. It may also be the case that MMP14 macrophages are performing a similar role in adult mouse hearts following injury but that another proregenerative process is absent. A failure to co-ordinate a multi-faceted regenerative response could in fact 480 be detrimental. Future research will be required to determine why MMP14 plays a positive pro-regenerative 481 role in adult zebrafish, and yet appears to exacerbate the damage associated with MI in mammals. response during cardiac regeneration in adult zebrafish. Although there are notable differences when 


\section{Materials and methods.}

\begin{tabular}{|c|c|c|c|c|}
\hline Sample & $\begin{array}{l}\text { unamputated } \\
\text { control }\end{array}$ & $\begin{array}{l}3 \text { days post- } \\
\text { amputation }\end{array}$ & $\begin{array}{l}7 \text { days post- } \\
\text { amputation }\end{array}$ & $\begin{array}{l}14 \text { days post- } \\
\text { amputation }\end{array}$ \\
\hline $\begin{array}{l}\text { number of hearts } \\
\text { pooled/dissociated }\end{array}$ & 10 & 10 & 10 & 10 \\
\hline number of cells & 1876 & 1565 & 3153 & 2475 cells \\
\hline $\begin{array}{l}\text { median genes per } \\
\text { cell }\end{array}$ & 1411 & 1339 & 1425 & 1450 \\
\hline
\end{tabular}

500 Cellular suspensions were loaded on a Chromium controller (10x Genomics, Pleasanton, CA, USA) to 501 generate single-cell Gel Beads-in-Emulsion (GEMs). Single-cell RNA-Seq libraries were prepared using

502 Chromium Single cell 3'RNA Gel Bead and Library Kit v3.1. GEM-RT was performed in a C1000 Touch 

$4^{\circ} \mathrm{C}$. After RT, GEMs were broken and the single-strand cDNA was cleaned up with DynaBeads MyOne Silane Beads (Thermo Fisher Scientific). cDNA was amplified using the C1000 Touch Thermal cycler with 96-DeepWell Reaction Module: $98^{\circ} \mathrm{C}$ for $3 \mathrm{~min}$; cycled 12 : $98^{\circ} \mathrm{C}$ for $15 \mathrm{~s}, 63^{\circ} \mathrm{C}$ for $20 \mathrm{~s}$, and $72^{\circ} \mathrm{C}$ for 1 min; $72^{\circ} \mathrm{C}$ for $1 \mathrm{~min}$; held at $4^{\circ} \mathrm{C}$. Amplified cDNA product was cleaned up with the SPRI select beads. Indexed sequencing libraries were constructed following these steps: (1) fragmentation, end-repair and Atailing and size selection with SPRIselect; (2) adapter ligation and cleanup with SPRIselect; (3) sample

510 index PCR and size selection with SPRIselect. The barcoded sequencing libraries were quantified by 511 quantitative PCR (KAPA Biosystems Library Quantification Kit for Illumina platforms). Sequencing 512 libraries were loaded at $300 \mathrm{pM}$ on an Illumina NovaSeq6000 using the following read length: 28 bp Read1, 5138 bp I7 Index, 91 bp Read2 (experiment 1), and 28 bp Read1, 10 bp I7 Index, 10 bp I5 Index, 87 bp Read2 514 (experiment 2).

515 Image analyses and base calling were performed using the NovaSeq Control Software and the Real-Time 516 Analysis component (Illumina). Demultiplexing was performed using the 10X Genomics software 517 Cellranger mkfastq (v3.1.0 for experiment 1 and v6.0.1 for experiment 2), a wrapper of Illumina's bcl2fastq 518 (v2.20). The quality of the raw data was assessed using FastQC (v0.11.8) from the Babraham Institute and 519 the Illumina software SAV (Sequencing Analysis Viewer). FastqScreen (v0.14.0) was used to estimate the 520 potential level of contamination.

521 Alignment, gene expression quantification and statistical analysis were performed using Cell Ranger count 522 on Danio rerio 's transcriptome GRCz11 (sequences and annotation were downloaded from Ensembl! on 523 July 24th, 2019). In order to discard ambient RNA falsely identified as cells, Cell Ranger count was run a 524 second time with the option --force-cells to force the number of cells to detect. Cell Ranger aggr was then used to combine each sample result into one single analysis. 
528 Datasets generated for this study: sequencing data have been deposited in the ArrayExpress database at

529 EMBL-EBI (www.ebi.ac.uk/arrayexpress) under accession code E-MTAB-10643.

530 Previously published datasets used for this study: E-MTAB-7376 (www.ebi.ac.uk/arrayexpress) from

531 Farbehi et al.(6); GSE153480 (www.ncbi.nlm.nih.gov/geo/) from Wang et al.(7).

Zebrafish transgenic lines and husbandry

534 Zebrafish were maintained under standardized conditions and experiments were conducted in accordance

535 with local approval (APAFIS\#4054-2016021116464098 v5) and the European Communities council

536 directive 2010/63/EU. Embryos were staged as described (67). The $T g(f l i l a: G F P) y l T g$ was provided by

537 the CMR[B] Centro de Medicina Regenerativa de Barcelona. Tg(gatal:dsred) and Tg(mpeg1.1:mCherry)

538 were provided by the Lutfalla Lab, University of Montpellier. $\operatorname{Tg}$ (colla2:mCherry) was provided by the

539 Mercader Lab, Bern University. Tg(eab2:[EGFP-T-mCherry] $)^{v 2295}$ was provided by the Chen Lab,

540 Vanderbilt University Medical Center. All larvae and adults were euthanised by administration of excess

541 anaesthetic (Tricaine).

542 Zebrafish cardiac regeneration.

543 All amputations were performed as described(2), in accordance with local approval (APAFIS\#4052). For 544 the scRNA-seq analysis we used 6 month old sibling offspring from an incross of $T g(\mathrm{cmlc} 2 a: G F P)$ which 545 were generated on an $\mathrm{AB}$ wildtype background.

548 All amputations were performed on Swiss-JL (Janvier labs) P1 neonatal mice as described(68), in 549 accordance with local approval (APAFIS\#1498-15516). 
552 EdU labelling was performed according to the manufacturers instructions (Click-iT EdU Kit C10337,

553 Molecular Probes). Amputated adult Tg(mpeg1.1:mCherry $\left.{ }^{+}\right)$were anesthetized in Tricaine then injected 554 with $50 \mu \mathrm{l}$ of a $240 \mu \mathrm{g} / \mathrm{ml}$ EdU solution daily.

Immunohistochemistry

557 Immunohistochemistry was performed on $10 \mu \mathrm{m}$ cryo-sections as previously described(59). The primary 558 antibodies used in this manuscript are anti-RFP (5F8 Chromotek), anti-GFP (GFP1020 Aves), anti-Acta2

559 GTX124505 Genetex), anti-RSPO1 (ab106556 Abcam), anti-Tal11 (abx339062 Abbexa), anti- $\alpha$

560 Sarcomeric actin (A2172 Sigma), anti-MMP14 (mbs422986 Mybiosource and GTX128198 Genetex), IB4

561 (Isolectin GS1B4) (I21413 Invitrogen), EdU labelling was performed according to the manufacturers

562 instructions (Click-iT EdU Kit C10337, Molecular Probes).

565 Acid Fuchsin-Orange G (AFOG) staining was performed on $10 \mu \mathrm{m}$ cryosections as previously described 566 (69).

569 An Olympus SZX16 fluorescence stereomicroscope fitted with a Jenoptik ProgRes CF Cool CCD

570 Microscope Camera was used for histological section imaging and a Leica TCS SP-8 confocal microscope

571 for imaging immunohistochemical labelling. Image acquisition and image analysis were performed on 572 workstations of the Montpellier RIO Imaging facility of Arnaud de Villeneuve. 
575 The DN tal construct and transgenic line were generated using the Tol2 Kit as described (70, 71). Dominant

576 negative zf tall was generated as described(36). For the Tg(fliEP:loxRFPlox:DNtal) construct the 5' entry

577 clone 478 p5Efli1ep was a gift from Nathan Lawson (Addgene plasmid \# 31160 ;

578 http://n2t.net/addgene:31160 ; RRID:Addgene_31160, the middle entry clone contained a floxed RFP stop

579 cassette amplified from pBOB-LRL-CBReGFPpA (a kind gift from Geoff Whal) and the 3' entry clone

580 contained zebrafish dominant negative tal1. For the Tg(fliEP:Ert2CreErt2) the 5' entry clone was p5Efli1ep

581 and the middle entry clone was pMEErt2CreErt2 as described(2).

582

583 Real-time quantitative RT-PCR

584 RNA was extracted from amputated/unamputated ventricles of $\mathrm{AB}$ wild type zebrafish and quantitative 585 PCR was performed using a Roche LightCycler 480 system as described (34). Primer sequences are 586 provided below.

588 MMP2 reverse- 5' AGGGTGCTCCATCTGAATTT 3'

MMP9 forward- 5' TTTGACGCCATCACTGAAAT 3'

MMP9 reverse- 5' TTCGCAGAGATCATGAAAGG 3'

MMP13a forward- 5' CTCAGAGCCCAGATGTTGAA 3'

592 MMP13a reverse- 5' CCTTCTCACCTTTGATCAGGA 3'

593 MMP14a forward- 5' CTCGCAAGTGTGTTTCTGGT 3'

594 MMP14a reverse- 5' TCACCAGGAGGAAGATACCC 3'

595 MMP14b forward- 5' GATATGAAACCTGAGGCATGG 3' 


\section{Chemical treatments}

599 Transgenes were expressed by inducing Cre mediated recombination with tamoxifen as described(2).

600 GM6001 (ab120845, Abcam) was dissolved in DMSO to reach a stock concentration of $10 \mathrm{mM}$ and then 601 split into $10 \mu \mathrm{l}$ aliquots and stored at $-20^{\circ} \mathrm{C}$. On the day of injection, the $10 \mu \mathrm{l}$ aliquot was added to $1 \mathrm{ml}$

602 1X PBS to reach a final concentration of $100 \mu \mathrm{M}$. This solution was administered daily i.p. Control groups 603 were administered daily with 1X PBS. NSC40520 (SML0518 Sigma) was diluted in DMSO to reach a stock 604 concentration of $10 \mathrm{Mm}$. 5 fish were placed in a beaker with 500ml of system water and 500 $\mu$ l of NSC40520 605 was added to reach a final concentration of $10 \mu \mathrm{M}$. Controls were placed in a beaker and 500 $\mu 1$ of DMSO 606 was added. The fish were left overnight then rinsed the next morning and returned back to the main 607 aquarium during the day. This was repeated for the duration of each experiment. All chemical treatments 608 were performed in accordance with local approval (APAFIS\#4054).

609

\section{Statistical analysis}

611 GraphPad prism was used to perform all statistical analysis. Details of statistical analysis are provided in 612 figures and figure legends. 


\section{References}

1. G. Savarese, L. H. Lund, Global Public Health Burden of Heart Failure. Cardiac failure review 3, 7-11 (2017); published online EpubApr (10.15420/cfr.2016:25:2).

2. C. Jopling, E. Sleep, M. Raya, M. Marti, A. Raya, J. C. Izpisua Belmonte, Zebrafish heart regeneration occurs by cardiomyocyte dedifferentiation and proliferation. Nature 464, 606-609 (2010); published online EpubMar 25 (10.1038/nature08899).

3. E. R. Porrello, A. I. Mahmoud, E. Simpson, J. A. Hill, J. A. Richardson, E. N. Olson, H. A. Sadek, Transient regenerative potential of the neonatal mouse heart. Science 331, 1078-1080 (2011); published online EpubFeb 25 (10.1126/science.1200708).

4. E. Forte, M. B. Furtado, N. Rosenthal, The interstitium in cardiac repair: role of the immunestromal cell interplay. Nature reviews. Cardiology 15, 601-616 (2018); published online EpubOct (10.1038/s41569-018-0077-x).

5. C. E. Fernandez, M. Bakovic, R. Karra, Endothelial Contributions to Zebrafish Heart Regeneration. Journal of cardiovascular development and disease 5, (2018); published online EpubDec 11 (10.3390/jcdd5040056).

6. N. Farbehi, R. Patrick, A. Dorison, M. Xaymardan, V. Janbandhu, K. Wystub-Lis, J. W. Ho, R. E. Nordon, R. P. Harvey, Single-cell expression profiling reveals dynamic flux of cardiac stromal, vascular and immune cells in health and injury. eLife 8, (2019); published online EpubMar 26 (10.7554/eLife.43882).

7. Z. Wang, M. Cui, A. M. Shah, W. Tan, N. Liu, R. Bassel-Duby, E. N. Olson, Cell-Type-Specific Gene Regulatory Networks Underlying Murine Neonatal Heart Regeneration at Single-Cell Resolution. Cell Rep 33, 108472 (2020); published online EpubDec 8 (10.1016/j.celrep.2020.108472).

8. R. J. Major, K. D. Poss, Zebrafish Heart Regeneration as a Model for Cardiac Tissue Repair. Drug discovery today. Disease models 4, 219-225 (2007)10.1016/j.ddmod.2007.09.002).

9. H. Sanchez-Iranzo, M. Galardi-Castilla, A. Sanz-Morejon, J. M. Gonzalez-Rosa, R. Costa, A. Ernst, J. Sainz de Aja, X. Langa, N. Mercader, Transient fibrosis resolves via fibroblast inactivation in the regenerating zebrafish heart. Proc Natl Acad Sci U S A 115, 4188-4193 (2018); published online EpubApr 17 (10.1073/pnas.1716713115).

10. F. Chablais, A. Jazwinska, The regenerative capacity of the zebrafish heart is dependent on TGFbeta signaling. Development 139, 1921-1930 (2012); published online EpubJun (10.1242/dev.078543).

11. J. Wang, R. Karra, A. L. Dickson, K. D. Poss, Fibronectin is deposited by injury-activated epicardial cells and is necessary for zebrafish heart regeneration. Developmental biology 382, 427435 (2013); published online EpubOct 15 (10.1016/j.ydbio.2013.08.012).

12. J. W. Godwin, R. Debuque, E. Salimova, N. A. Rosenthal, Heart regeneration in the salamander relies on macrophage-mediated control of fibroblast activation and the extracellular landscape. NPJ Regenerative medicine 2, (2017)10.1038/s41536-017-0027-y).

13. F. C. Simoes, T. J. Cahill, A. Kenyon, D. Gavriouchkina, J. M. Vieira, X. Sun, D. Pezzolla, C. Ravaud, E. Masmanian, M. Weinberger, S. Mayes, M. E. Lemieux, D. N. Barnette, M. GunadasaRohling, R. M. Williams, D. R. Greaves, L. A. Trinh, S. E. Fraser, S. L. Dallas, R. P. Choudhury, T. Sauka-Spengler, P. R. Riley, Macrophages directly contribute collagen to scar formation during zebrafish heart regeneration and mouse heart repair. Nat Commun 11, 600 (2020); published online EpubJan 30 (10.1038/s41467-019-14263-2). 
663

664

665

666

667

668

669

670

671

672

673

674

675

676

677

678

679

680

681

682

683

684

685

686

687

688

689

690

691

692

693

694

695

696

697

698

699

700

701

702

703

704

705

706

707

708

709

710

711

712

14. L. Bevan, Z. W. Lim, B. Venkatesh, P. R. Riley, P. Martin, R. J. Richardson, Specific macrophage populations promote both cardiac scar deposition and subsequent resolution in adult zebrafish. Cardiovasc Res 116, 1357-1371 (2020); published online EpubJun 1 (10.1093/cvr/cvz221).

15. A. B. Aurora, E. R. Porrello, W. Tan, A. I. Mahmoud, J. A. Hill, R. Bassel-Duby, H. A. Sadek, E. $\mathrm{N}$. Olson, Macrophages are required for neonatal heart regeneration. The Journal of clinical investigation 124, 1382-1392 (2014); published online EpubMar (10.1172/JCI72181).

16. C. Patra, Z. Kontarakis, H. Kaur, A. Rayrikar, D. Mukherjee, D. Y. R. Stainier, The zebrafish ventricle: A hub of cardiac endothelial cells for in vitro cell behavior studies. Scientific reports 7, 2687 (2017); published online EpubJun 2 (10.1038/s41598-017-02461-1).

17. A. R. Pinto, A. Ilinykh, M. J. Ivey, J. T. Kuwabara, M. L. D'Antoni, R. Debuque, A. Chandran, L. Wang, K. Arora, N. A. Rosenthal, M. D. Tallquist, Revisiting Cardiac Cellular Composition. Circ Res 118, 400-409 (2016); published online EpubFeb 5 (10.1161/CIRCRESAHA.115.307778).

18. F. Sommer, V. Torraca, S. M. Kamel, A. Lombardi, A. H. Meijer, Frontline Science: Antagonism between regular and atypical $\mathrm{Cxcr} 3$ receptors regulates macrophage migration during infection and injury in zebrafish. Journal of leukocyte biology 107, 185-203 (2020); published online EpubFeb (10.1002/JLB.2HI0119-006R).

19. S. Epelman, K. J. Lavine, A. E. Beaudin, D. K. Sojka, J. A. Carrero, B. Calderon, T. Brija, E. L. Gautier, S. Ivanov, A. T. Satpathy, J. D. Schilling, R. Schwendener, I. Sergin, B. Razani, E. C. Forsberg, W. M. Yokoyama, E. R. Unanue, M. Colonna, G. J. Randolph, D. L. Mann, Embryonic and adult-derived resident cardiac macrophages are maintained through distinct mechanisms at steady state and during inflammation. Immunity 40, 91-104 (2014); published online EpubJan 16 (10.1016/j.immuni.2013.11.019).

20. K. J. Mould, N. D. Jackson, P. M. Henson, M. Seibold, W. J. Janssen, Single cell RNA sequencing identifies unique inflammatory airspace macrophage subsets. JCI insight 4, (2019); published online EpubMar 7 (10.1172/jci.insight.126556).

21. A. Das, V. Sudhahar, G. F. Chen, H. W. Kim, S. W. Youn, L. Finney, S. Vogt, J. Yang, J. Kweon, B. Surenkhuu, M. Ushio-Fukai, T. Fukai, Endothelial Antioxidant-1: a Key Mediator of Copperdependent Wound Healing in vivo. Scientific reports 6, 33783 (2016); published online EpubSep $26(10.1038 /$ srep33783).

22. T. Moore-Morris, N. Guimaraes-Camboa, I. Banerjee, A. C. Zambon, T. Kisseleva, A. Velayoudon, W. B. Stallcup, Y. Gu, N. D. Dalton, M. Cedenilla, R. Gomez-Amaro, B. Zhou, D. A. Brenner, K. L. Peterson, J. Chen, S. M. Evans, Resident fibroblast lineages mediate pressure overload-induced cardiac fibrosis. The Journal of clinical investigation 124, 2921-2934 (2014); published online EpubJul (10.1172/JCI74783).

23. A. J. Moyes, R. S. Khambata, I. Villar, K. J. Bubb, R. S. Baliga, N. G. Lumsden, F. Xiao, P. J. Gane, A. S. Rebstock, R. J. Worthington, M. I. Simone, F. Mota, F. Rivilla, S. Vallejo, C. Peiro, C. F. Sanchez Ferrer, S. Djordjevic, M. J. Caulfield, R. J. MacAllister, D. L. Selwood, A. Ahluwalia, A. J. Hobbs, Endothelial C-type natriuretic peptide maintains vascular homeostasis. The Journal of clinical investigation 124, 4039-4051 (2014); published online EpubSep (10.1172/JCI74281).

24. M. D. Tallquist, J. D. Molkentin, Redefining the identity of cardiac fibroblasts. Nature reviews. Cardiology 14, 484-491 (2017); published online EpubAug (10.1038/nrcardio.2017.57).

25. M. Sande-Melon, I. J. Marques, M. Galardi-Castilla, X. Langa, M. Perez-Lopez, M. A. Botos, H. Sanchez-Iranzo, G. Guzman-Martinez, D. M. Ferreira Francisco, D. Pavlinic, V. Benes, R. Bruggmann, N. Mercader, Adult sox10(+) Cardiomyocytes Contribute to Myocardial Regeneration in the Zebrafish. Cell Rep 29, 1041-1054 e1045 (2019); published online EpubOct 22 (10.1016/j.celrep.2019.09.041).

26. C. Humeres, N. G. Frangogiannis, Fibroblasts in the Infarcted, Remodeling, and Failing Heart. JACC. Basic to translational science 4, 449-467 (2019); published online EpubJun (10.1016/j.jacbts.2019.02.006). 
27. S. Xu, C. Liu, F. Xie, L. Tian, S. H. Manno, F. A. M. Manno, 3rd, S. Fallah, B. Pelster, G. Tse, S. H. Cheng, Excessive inflammation impairs heart regeneration in zebrafish breakdance mutant after cryoinjury. Fish \& shellfish immunology 89, 117-126 (2019); published online EpubJun (10.1016/j.fsi.2019.03.058).

28. C. L. Lien, M. Schebesta, S. Makino, G. J. Weber, M. T. Keating, Gene expression analysis of zebrafish heart regeneration. PLoS Biol 4, e260 (2006); published online EpubAug (10.1371/journal.pbio.0040260).

29. J. Koth, X. Wang, A. C. Killen, W. T. Stockdale, H. G. Potts, A. Jefferson, F. Bonkhofer, P. R. Riley, R. K. Patient, B. Gottgens, M. T. M. Mommersteeg, Runx1 promotes scar deposition and inhibits myocardial proliferation and survival during zebrafish heart regeneration. Development 147, (2020); published online EpubApr 27 (10.1242/dev.186569).

30. R. Marin-Juez, M. Marass, S. Gauvrit, A. Rossi, S. L. Lai, S. C. Materna, B. L. Black, D. Y. Stainier, Fast revascularization of the injured area is essential to support zebrafish heart regeneration. Proc Natl Acad Sci U S A 113, 11237-11242 (2016); published online EpubOct 4 (10.1073/pnas.1605431113).

31. K. Kikuchi, J. E. Holdway, R. J. Major, N. Blum, R. D. Dahn, G. Begemann, K. D. Poss, Retinoic acid production by endocardium and epicardium is an injury response essential for zebrafish heart regeneration. Dev Cell 20, 397-404 (2011); published online EpubMar 15 (10.1016/j.devcel.2011.01.010).

32. J. Munch, D. Grivas, A. Gonzalez-Rajal, R. Torregrosa-Carrion, J. L. de la Pompa, Notch signalling restricts inflammation and serpinel expression in the dynamic endocardium of the regenerating zebrafish heart. Development 144, 1425-1440 (2017); published online EpubApr 15 (10.1242/dev.143362).

33. S. De Val, Key transcriptional regulators of early vascular development. Arterioscler Thromb Vasc Biol 31, 1469-1475 (2011); published online EpubJul (10.1161/ATVBAHA.110.221168).

34. C. Jopling, G. Sune, A. Faucherre, C. Fabregat, J. C. Izpisua Belmonte, Hypoxia induces myocardial regeneration in zebrafish. Circulation 126, 3017-3027 (2012); published online EpubDec 18 (10.1161/CIRCULATIONAHA.112.107888).

35. V. S. Stanulovic, P. Cauchy, S. A. Assi, M. Hoogenkamp, LMO2 is required for TAL1 DNA binding activity and initiation of definitive haematopoiesis at the haemangioblast stage. Nucleic acids research 45, 9874-9888 (2017); published online EpubSep 29 (10.1093/nar/gkx573).

36. P. D. Aplan, K. Nakahara, S. H. Orkin, I. R. Kirsch, The SCL gene product: a positive regulator of erythroid differentiation. The EMBO journal 11, 4073-4081 (1992); published online EpubNov (

37. C. Brosseau, L. Colas, A. Magnan, S. Brouard, CD9 Tetraspanin: A New Pathway for the Regulation of Inflammation? Frontiers in immunology 9, 2316 (2018)10.3389/fimmu.2018.02316).

38. L. Franchi, T. Eigenbrod, R. Munoz-Planillo, G. Nunez, The inflammasome: a caspase-1-activation platform that regulates immune responses and disease pathogenesis. Nature immunology 10, 241247 (2009); published online EpubMar (10.1038/ni.1703).

39. C. Qiao, N. Yin, H. Y. Gu, J. L. Zhu, J. H. Ding, M. Lu, G. Hu, Atp13a2 Deficiency Aggravates Astrocyte-Mediated Neuroinflammation via NLRP3 Inflammasome Activation. CNS neuroscience \& therapeutics 22, 451-460 (2016); published online EpubJun (10.1111/cns.12514).

40. V. Chitu, E. R. Stanley, Colony-stimulating factor-1 in immunity and inflammation. Current opinion in immunology 18, 39-48 (2006); published online EpubFeb (10.1016/j.coi.2005.11.006).

41. D. M. Mitchell, C. Sun, S. S. Hunter, D. D. New, D. L. Stenkamp, Regeneration associated transcriptional signature of retinal microglia and macrophages. Scientific reports 9, 4768 (2019); published online EpubMar 18 (10.1038/s41598-019-41298-8).

42. H. B. Sager, M. Hulsmans, K. J. Lavine, M. B. Moreira, T. Heidt, G. Courties, Y. Sun, Y. Iwamoto, B. Tricot, O. F. Khan, J. E. Dahlman, A. Borodovsky, K. Fitzgerald, D. G. Anderson, R. Weissleder, P. Libby, F. K. Swirski, M. Nahrendorf, Proliferation and Recruitment Contribute to 
804

805

806

807

808

809

810

811

812

813

Myocardial Macrophage Expansion in Chronic Heart Failure. Circ Res 119, 853-864 (2016); published online EpubSep 16 (10.1161/CIRCRESAHA.116.309001).

43. T. B. Levring, M. Kongsbak-Wismann, A. K. O. Rode, F. A. H. Al-Jaberi, D. V. Lopez, O. Met, A. Woetmann, C. M. Bonefeld, N. Odum, C. Geisler, Tumor necrosis factor induces rapid downregulation of TXNIP in human T cells. Scientific reports 9, 16725 (2019); published online EpubNov 13 (10.1038/s41598-019-53234-x).

44. Y. Ma, A. J. Mouton, M. L. Lindsey, Cardiac macrophage biology in the steady-state heart, the aging heart, and following myocardial infarction. Translational research : the journal of laboratory and clinical medicine 191, 15-28 (2018); published online EpubJan (10.1016/j.trsl.2017.10.001).

45. F. K. Swirski, M. Nahrendorf, Leukocyte behavior in atherosclerosis, myocardial infarction, and heart failure. Science 339, 161-166 (2013); published online EpubJan 11 (10.1126/science.1230719).

46. G. Viswanathan, D. M. Tobin, Macrophage ACKRobatics: An atypical Cxcr3 keeps macrophages in check. Journal of leukocyte biology 107, 171-173 (2020); published online EpubFeb (10.1002/JLB.4CE1019-384R).

47. L. Gamba, A. Amin-Javaheri, J. Kim, D. Warburton, C. L. Lien, Collagenolytic Activity Is Associated with Scar Resolution in Zebrafish Hearts after Cryoinjury. Journal of cardiovascular development and disease 4, (2017); published online EpubFeb 24 (10.3390/jcdd4010002).

48. S. Bai, R. Thummel, A. R. Godwin, H. Nagase, Y. Itoh, L. Li, R. Evans, J. McDermott, M. Seiki, M. P. Sarras, Jr., Matrix metalloproteinase expression and function during fin regeneration in zebrafish: analysis of MT1-MMP, MMP2 and TIMP2. Matrix biology : journal of the International Society for Matrix Biology 24, 247-260 (2005); published online EpubJun (10.1016/j.matbio.2005.03.007).

49. V. Vinarsky, D. L. Atkinson, T. J. Stevenson, M. T. Keating, S. J. Odelberg, Normal newt limb regeneration requires matrix metalloproteinase function. Developmental biology 279, 86-98 (2005); published online EpubMar 1 (10.1016/j.ydbio.2004.12.003).

50. I. S. Park, W. S. Kim, Modulation of gelatinase activity correlates with the dedifferentiation profile of regenerating salamander limbs. Mol Cells 9, 119-126 (1999); published online EpubApr 30 (

51. A. G. Remacle, V. S. Golubkov, S. A. Shiryaev, R. Dahl, J. L. Stebbins, A. V. Chernov, A. V. Cheltsov, M. Pellecchia, A. Y. Strongin, Novel MT1-MMP small-molecule inhibitors based on insights into hemopexin domain function in tumor growth. Cancer research 72, 2339-2349 (2012); published online EpubMay 1 (10.1158/0008-5472.CAN-11-4149).

52. M. Stawowczyk, M. D. Wellenstein, S. B. Lee, S. Yomtoubian, A. Durrans, H. Choi, N. Narula, N. K. Altorki, D. Gao, V. Mittal, Matrix Metalloproteinase 14 promotes lung cancer by cleavage of Heparin-Binding EGF-like Growth Factor. Neoplasia 19, 55-64 (2017); published online EpubFeb (10.1016/j.neo.2016.11.005).

53. H. Hara, N. Takeda, I. Komuro, Pathophysiology and therapeutic potential of cardiac fibrosis. Inflammation and regeneration 37, 13 (2017)10.1186/s41232-017-0046-5).

54. S. Allanki, B. Strilic, L. Scheinberger, Y. L. Onderwater, A. Marks, S. Gunther, J. Preussner, K. Kikhi, M. Looso, D. Y. R. Stainier, S. Reischauer, Interleukin-11 signaling promotes cellular reprogramming and limits fibrotic scarring during tissue regeneration. Science advances 7, eabg6497 (2021); published online EpubSep 10 (10.1126/sciadv.abg6497).

55. M. M. Ibrahim, L. Chen, J. E. Bond, M. A. Medina, L. Ren, G. Kokosis, A. M. Selim, H. Levinson, Myofibroblasts contribute to but are not necessary for wound contraction. Laboratory investigation; a journal of technical methods and pathology 95, 1429-1438 (2015); published online EpubDec (10.1038/labinvest.2015.116).

56. J. A. Schumacher, J. Bloomekatz, Z. V. Garavito-Aguilar, D. Yelon, tal1 Regulates the formation of intercellular junctions and the maintenance of identity in the endocardium. Developmental biology 383, 214-226 (2013); published online EpubNov 15 (10.1016/j.ydbio.2013.09.019).

57. T. Tang, Y. Shi, S. R. Opalenik, D. M. Brantley-Sieders, J. Chen, J. M. Davidson, S. J. Brandt, Expression of the TAL1/SCL transcription factor in physiological and pathological vascular 
processes. The Journal of pathology 210, 121-129 (2006); published online EpubSep (10.1002/path.2028).

58. I. Chrifi, D. Hermkens, M. M. Brandt, C. G. M. van Dijk, P. E. Burgisser, R. Haasdijk, J. Pei, E. H. M. van de Kamp, C. Zhu, L. Blonden, J. M. Kros, D. J. Duncker, H. J. Duckers, C. Cheng, Cgnl1, an endothelial junction complex protein, regulates GTPase mediated angiogenesis. Cardiovasc Res 113, 1776-1788 (2017); published online EpubDec 1 (10.1093/cvr/cvx175).

59. S. L. Lai, R. Marin-Juez, P. L. Moura, C. Kuenne, J. K. H. Lai, A. T. Tsedeke, S. Guenther, M. Looso, D. Y. Stainier, Reciprocal analyses in zebrafish and medaka reveal that harnessing the immune response promotes cardiac regeneration. eLife 6, (2017); published online EpubJun 20 (10.7554/eLife.25605).

60. K. J. Lavine, S. Epelman, K. Uchida, K. J. Weber, C. G. Nichols, J. D. Schilling, D. M. Ornitz, G. J. Randolph, D. L. Mann, Distinct macrophage lineages contribute to disparate patterns of cardiac recovery and remodeling in the neonatal and adult heart. Proc Natl Acad Sci U S A 111, 1602916034 (2014); published online EpubNov 11 (10.1073/pnas.1406508111).

61. V. Torraca, C. Cui, R. Boland, J. P. Bebelman, A. M. van der Sar, M. J. Smit, M. Siderius, H. P. Spaink, A. H. Meijer, The CXCR3-CXCL11 signaling axis mediates macrophage recruitment and dissemination of mycobacterial infection. Disease models \& mechanisms 8, 253-269 (2015); published online EpubMar (10.1242/dmm.017756).

62. G. C. Koenig, R. G. Rowe, S. M. Day, F. Sabeh, J. J. Atkinson, K. R. Cooke, S. J. Weiss, MT1MMP-dependent remodeling of cardiac extracellular matrix structure and function following myocardial infarction. The American journal of pathology 180, 1863-1878 (2012); published online EpubMay (10.1016/j.ajpath.2012.01.022).

63. R. Iwamoto, E. Mekada, ErbB and HB-EGF signaling in heart development and function. Cell structure and function 31, 1-14 (2006)10.1247/csf.31.1).

64. N. Tanaka, K. Masamura, M. Yoshida, M. Kato, Y. Kawai, I. Miyamori, A role of heparin-binding epidermal growth factor-like growth factor in cardiac remodeling after myocardial infarction. Biochemical and biophysical research communications 297, 375-381 (2002); published online EpubSep 20 (10.1016/s0006-291x(02)02197-6).

65. H. Ushikoshi, T. Takahashi, X. Chen, N. C. Khai, M. Esaki, K. Goto, G. Takemura, R. Maruyama, S. Minatoguchi, T. Fujiwara, S. Nagano, K. Yuge, T. Kawai, Y. Murofushi, H. Fujiwara, K. Kosai, Local overexpression of HB-EGF exacerbates remodeling following myocardial infarction by activating noncardiomyocytes. Laboratory investigation; a journal of technical methods and pathology 85, 862-873 (2005); published online EpubJul (10.1038/labinvest.3700282).

66. L. Alonso-Herranz, A. Sahun-Espanol, A. Paredes, P. Gonzalo, P. Gkontra, V. Nunez, C. Clemente, M. Cedenilla, M. Villalba-Orero, J. Inserte, D. Garcia-Dorado, A. G. Arroyo, M. Ricote, Macrophages promote endothelial-to-mesenchymal transition via MT1-MMP/TGFbeta1 after myocardial infarction. eLife 9, (2020); published online EpubOct 16 (10.7554/eLife.57920).

67. C. B. Kimmel, W. W. Ballard, S. R. Kimmel, B. Ullmann, T. F. Schilling, Stages of embryonic development of the zebrafish. Developmental dynamics : an official publication of the American Association of Anatomists 203, 253-310 (1995); published online EpubJul (10.1002/aja.1002030302).

68. A. I. Mahmoud, E. R. Porrello, W. Kimura, E. N. Olson, H. A. Sadek, Surgical models for cardiac regeneration in neonatal mice. Nature protocols 9, 305-311 (2014); published online EpubFeb (10.1038/nprot.2014.021).

69. K. D. Poss, L. G. Wilson, M. T. Keating, Heart regeneration in zebrafish. Science 298, 2188-2190 (2002); published online EpubDec 13 (10.1126/science.1077857).

70. K. M. Kwan, E. Fujimoto, C. Grabher, B. D. Mangum, M. E. Hardy, D. S. Campbell, J. M. Parant, H. J. Yost, J. P. Kanki, C. B. Chien, The Tol2kit: a multisite gateway-based construction kit for Tol2 transposon transgenesis constructs. Developmental dynamics : an official publication of the American Association of Anatomists 236, 3088-3099 (2007); published online EpubNov ( 
864 71. C. Jopling, E. Sleep, M. Raya, M. Marti, A. Raya, J. C. Belmonte, Zebrafish heart regeneration

865

866

867

\section{Acknowledgements.}

869 We acknowledge the imaging facility MRI, member of the national infrastructure France-BioImaging 870 infrastructure supported by the French National Research Agency (ANR-10-INBS-04, «Investments for the

871 future»). Montpellier Genomix (MGX). MGX acknowledges financial support from France Génomique 872 National infrastrusture, funded as part of "Investissement d'Avenir" program managed by Agence National 873 pour la Recherche (contract ANR-10-INBS-09). The Jopling lab is part of the Laboratory of Excellence Ion 874 Channel Science and Therapeutics supported by a grant from the ANR. Work in the Jopling lab is supported 875 by a grant from the "la Fondation Leducq" and from the ANR (contract ANR-20-CE14-003 MetabOx876 Heart). 
bioRxiv preprint doi: https://doi.org/10.1101/2021.10.25.465720; this version posted October 26, 2021. The copyright holder for this preprint (which was not certified by peer review) is the author/funder. All rights reserved. No reuse allowed without permission.

Figure 1

A

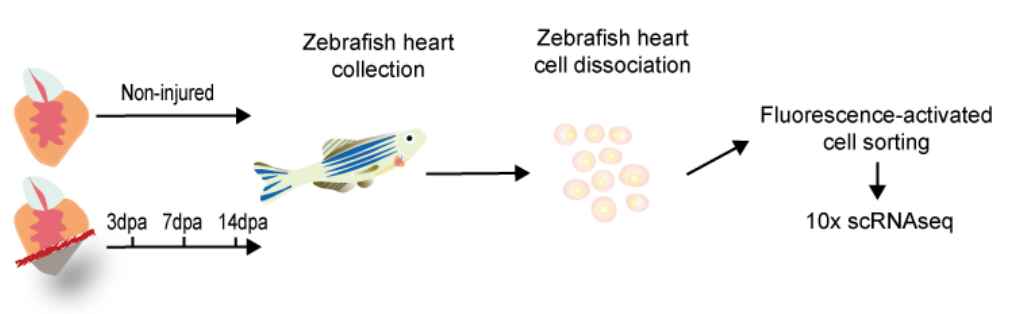

B

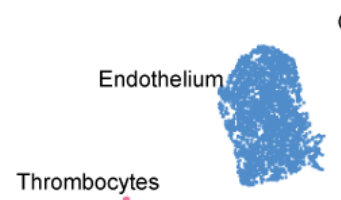

Cardiac neural crest-derived cells

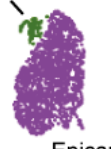

Epicardium/EPDCs
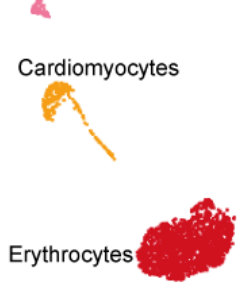

Lymphoid
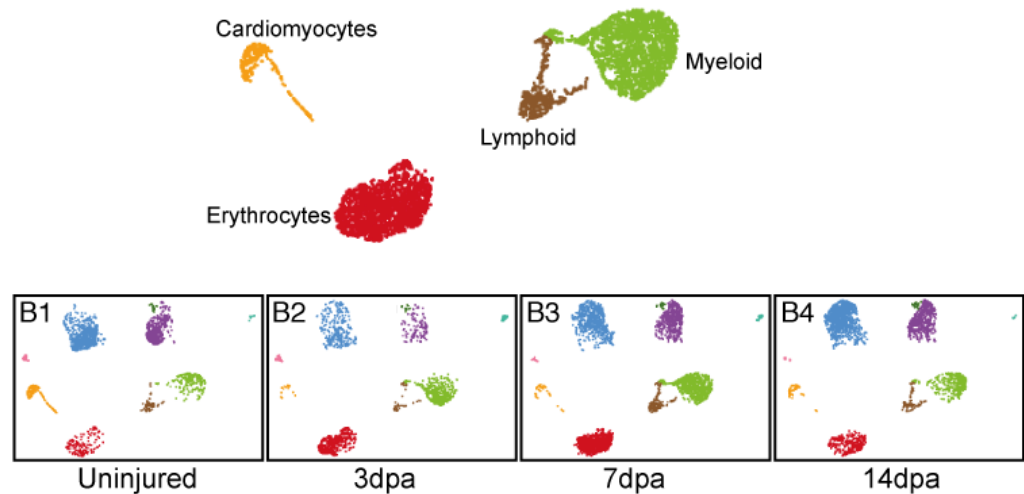

Uninjured

D

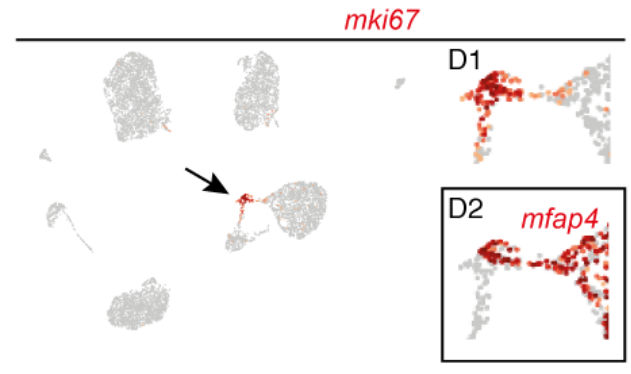

E

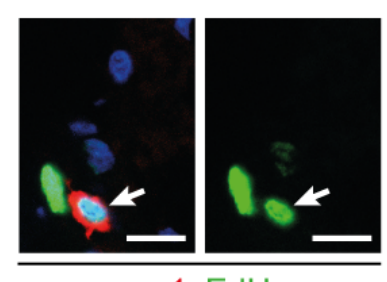

mpeg1 EdU
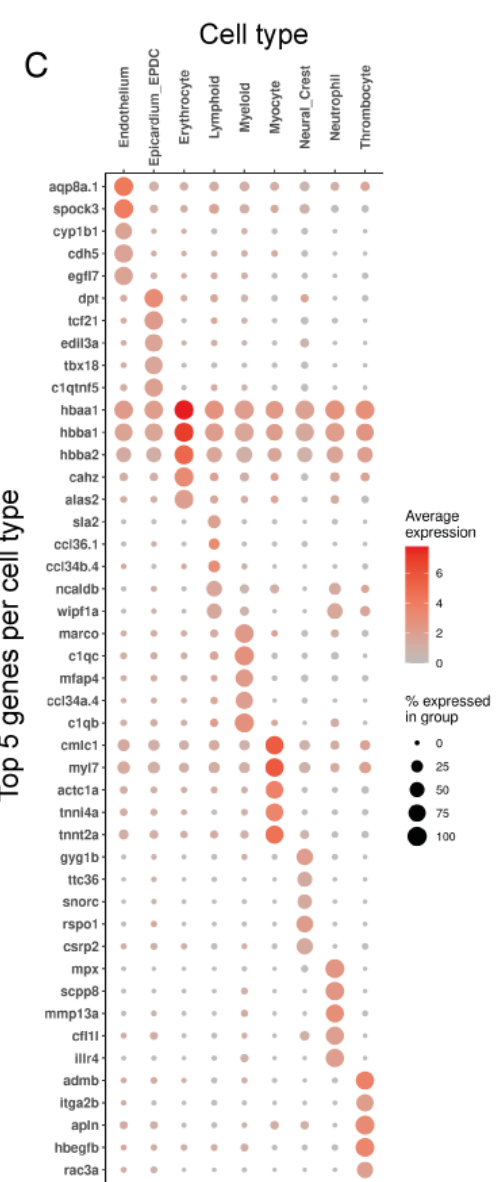

F

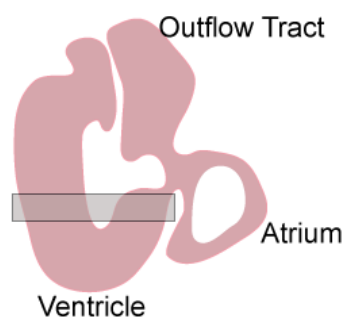

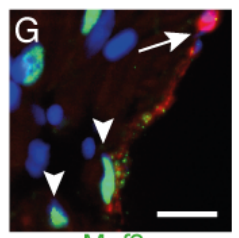

Mef2c

col1a2:mCherry

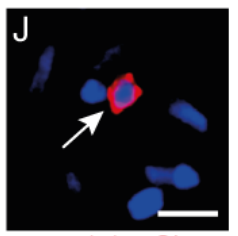

mpeg1.1:mCherry

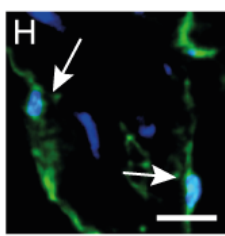

fli1a:GFP

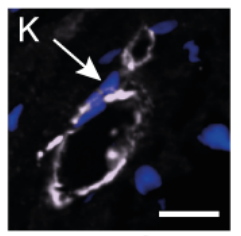

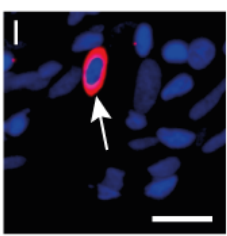

gata1:RFP

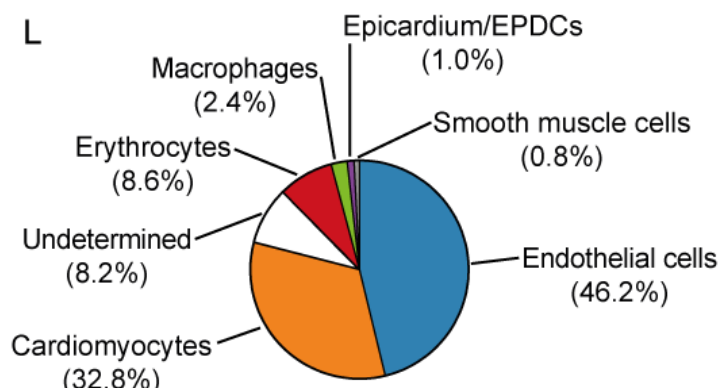

(32.8\%) 

seq pipeline. (B) UMAP clusters of the different populations of cells identified in zebrafish hearts. (B1-4) UMAP clusters in uninjured, 3 days post amputation (dpa), 7 dpa and 14 dpa. (C) A DotPlot showing the

882 cells based on the expression of mki67, black arrow points to the cluster which is shown at higher 883 magnification (D1). (D2) The proliferating cluster contains $\mathrm{mfap}^{+}$myeloid (macrophages) and $\mathrm{mfap}^{-}$ 884 lymphoid cells. (E) IHC of EdU labelled 3dpa regenerating zebrafish hearts. Mpeg1 (red) labels 885 macrophages, EdU (green) labels proliferating cells, DAPI (blue) (scale bar 10 $\mu \mathrm{m}$ ). (F) Diagram of an adult 886 zebrafish heart, the shaded rectangle indicates the area which was used to count the different cell type. (G887 K) Examples of IHC images used to count the different cell types. Cardiomyocytes, Mef2c (green). 888 Fibroblasts, Col1a2:mCherry (red). Endothelium, Fli1a:GFP (green). Erythrocytes, Gata1:RFP (red).

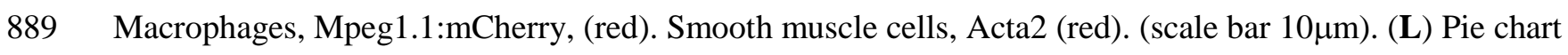
890 representing the proportions of different cell types present within the uninjured adult zebrafish ventricle.

891 The average percentage of cells positive for each cell-type specific marker were identified among at least 892800 cells per heart, with 5 hearts analysed for each marker. 
Figure 2

A

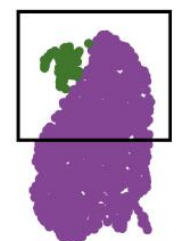

Epicardium_EPDC

- Neural Crest

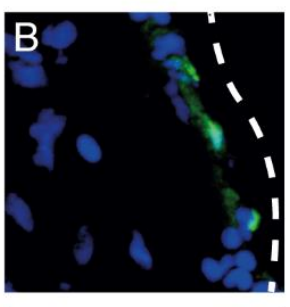

DAPI Rspo1
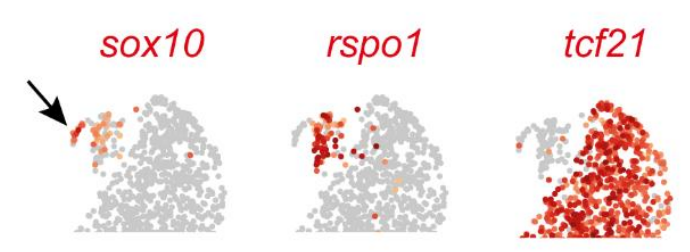

pdgfra

tbx18
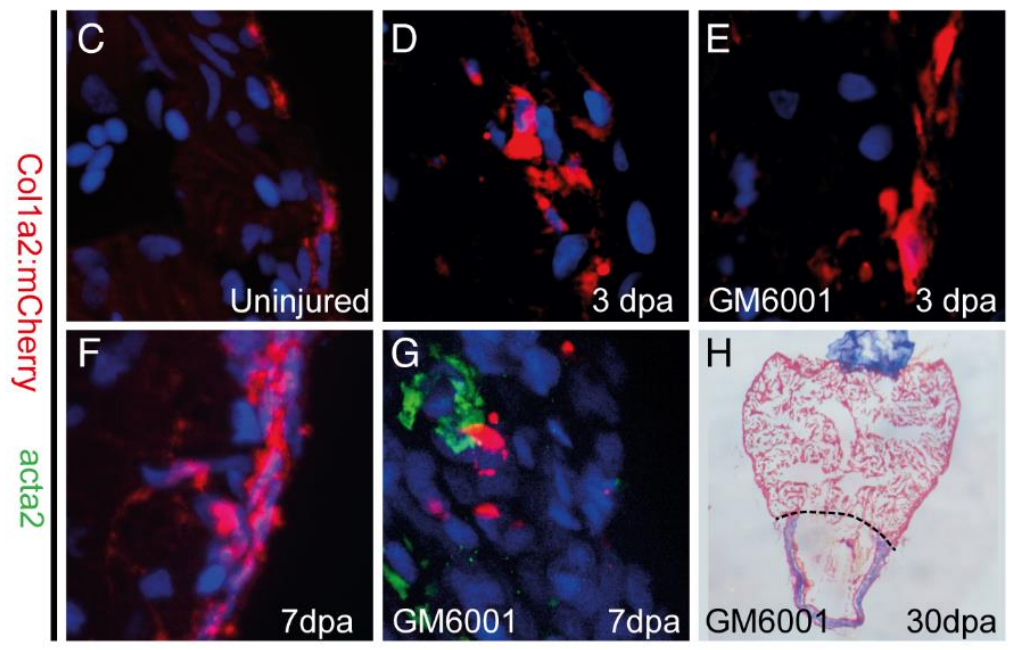
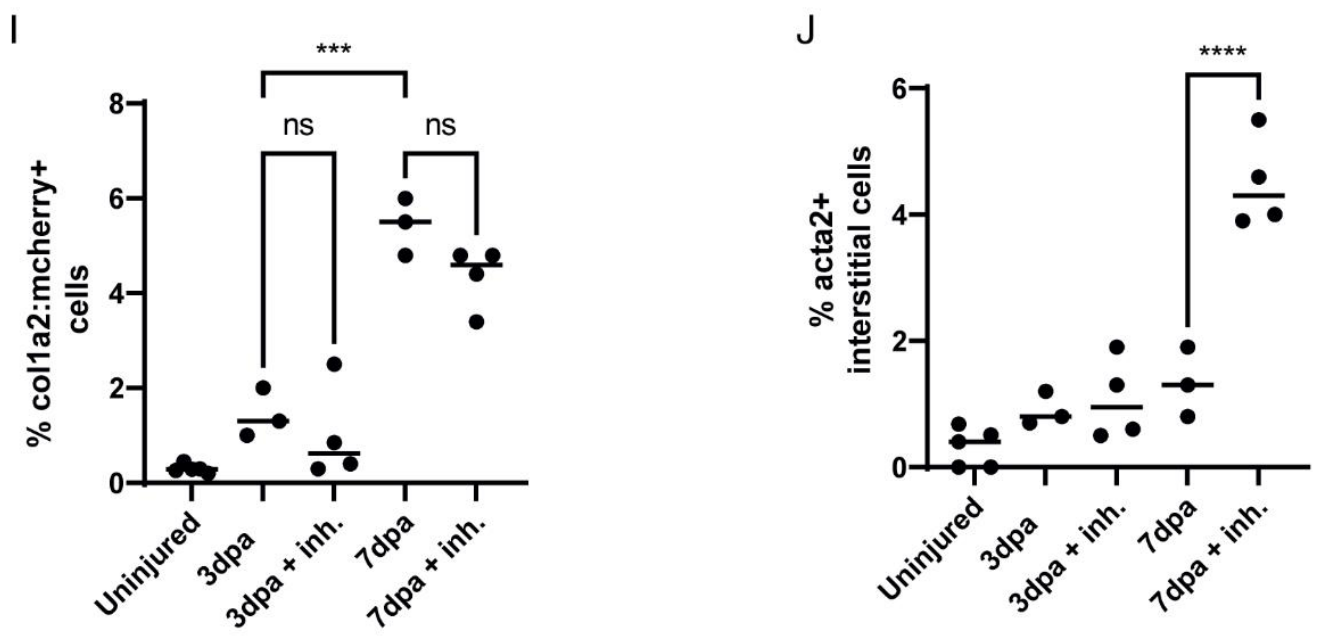

894 Figure 2. Mesenchymal lineages and cardiac fibroblast activation in the regenerating zebrafish heart.

895 (A) UMAP plot indicating the epicardial (purple) and neural crest (green) clusters. The black square delineates the area depicted in the subsequent UMAP plots. UMAP plots depicting the relative expression 
$898 p d g f r a($ Epicardium/EPDC) and $t b x 18$ (Epicardium/EPDC). Note that $r s p o 1$ is restricted to the neural crest 899 cluster and does not segregate with either $t c f 21$ or $t b x 18$. (B) IHC image of a $T g($ colla2:mCherry) zebrafish 900 heart labelled with a RSPO1 antibody (green). (C-G) IHC images of Tg(colla2: $m$ Cherry) zebrafish hearts 901 showing Col1a1:mCherry (red, fibroblasts) and Acta2 (green) in uninjured (C), 3dpa (D), 3dpa inhibitor 902 treated (E), 7dpa (F) and 7dpa inhibitor treated hearts $(\mathbf{G})$. Note the absence of Col1a2:mCherry and Acta2 903 co-localisation. Although Acta2 (green) is present in the 7dpa inhibitor treated sample this does not co904 localise with Col1a2:mCherry (H) AFOG staining of a MMP inhibitor (GM6001) treated zebrafish heart at 905 30dpa, black dashed line indicates the plane of amputation. Note the presence of a large fibrin 906 (red)/collagen(blue) scar. (I) Graph showing the percentage of cells, from an average of 650 cells per heart, 907 that were Col1a2:mcherry ${ }^{+}$in the different experimental conditions. (J) Graph showing the percentage of 908 interstitial cells, from an average of 650 cells per heart, that produced Acta 2 in the different experimental 909 conditions. Note that inhibitor treatment significantly increases the number of Acta2 cells. P values were 910 calculated by 1 -way ANOVA. $* * * P<0.001$, **** $P<0.0001$; ns, non-significant. 
Figure 3

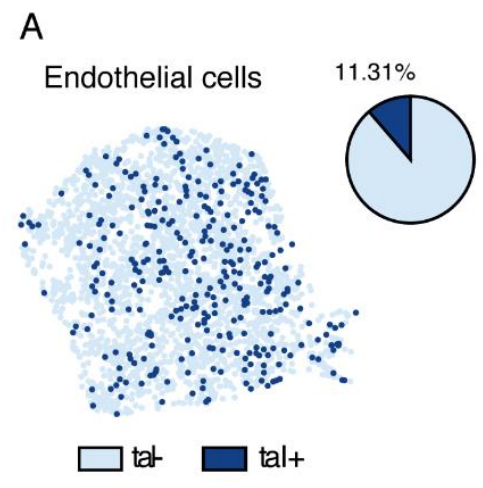

B

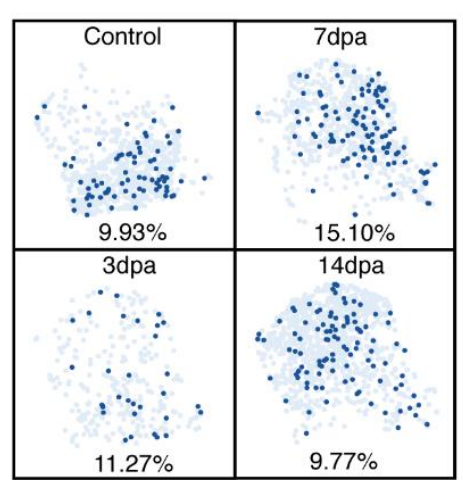

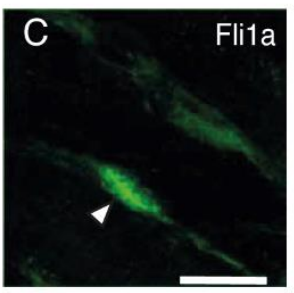

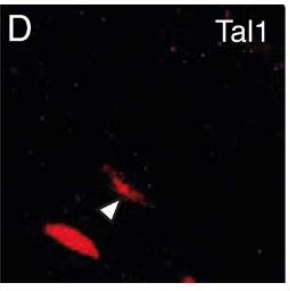

G

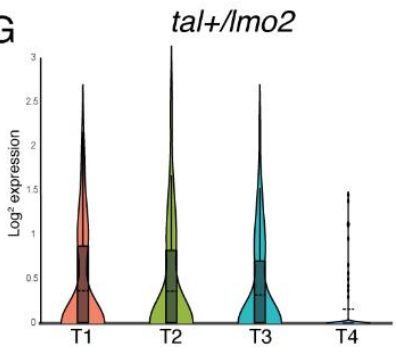

$\mathrm{H}$

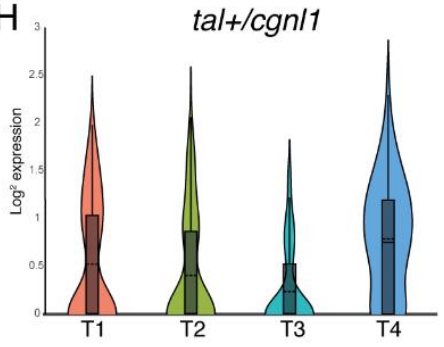

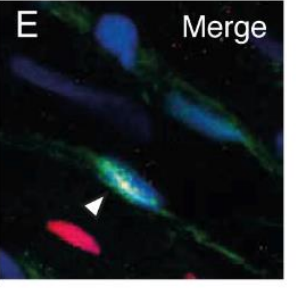

I

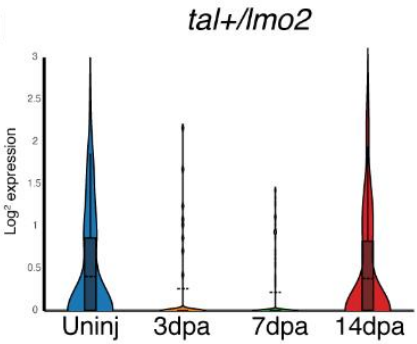

$\mathrm{J}$

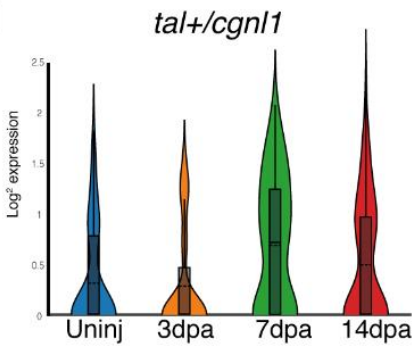

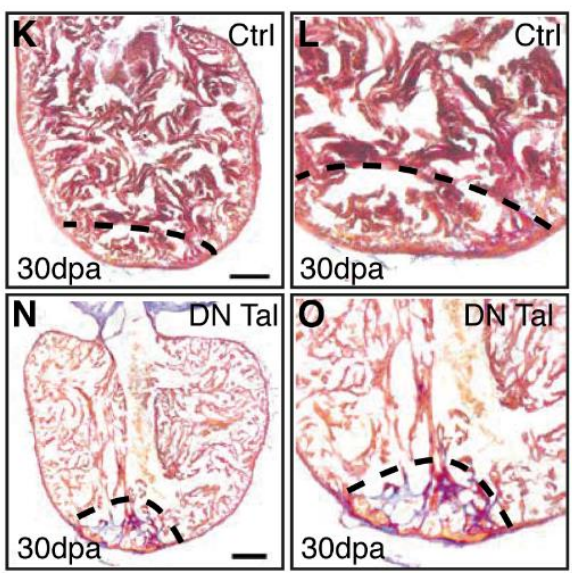

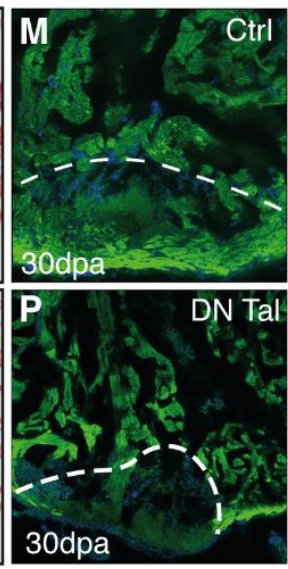


912 Figure 3. Tal1 is a regulator of the endothelial regenerative response. (A) UMAP plot of the endothelial

913 cell cluster (light blue dots) showing tal1 is expressed in a subset of these cells (dark blue dots). The pie

914 chart indicates the total proportion of endothelial cells which express tall. (B) UMAP plots of the

915 endothelial population at different stages of regeneration (control/unamputated, 3dpa, 7dpa and 14dpa). The

916 proportion of tall expressing endothelial cells (dark blue dots) is given as a percentage underneath each

917 UMAP plot. (C-E) IHC for endothelial cells (C, green), Tal1 (D, red), merged image (E) (scale bar 10 1 m).

918 (F) Analysis of tal1 expressing cells. UMAP plot of re-clustered tall expressing cells. Coloured boxes

919 represent the different clusters within this population (T1-T4) (G-J). Violin plots indicate the expression of

920 either $l m o 2$ or cgnll in each cluster $(\mathbf{G}, \mathbf{H})$ and at each time point during regeneration $(\mathbf{I}, \mathbf{J})$. (K) AFOG

921 staining of a control heart at 30dpa, black dashed line indicates the plane of amputation (scale bar $200 \mu \mathrm{m}$ ).

922 (L) The same image at higher magnification. (M) IHC of the same heart at 30dpa labelled with an anti-

$923 \alpha$ sarcomeric actin antibody (green). (N) AFOG staining of a DN Tal1 expressing heart at 30dpa, black

924 dashed line indicates the plane of amputation (scale bar $200 \mu \mathrm{m}$ ). (O) The same image at higher

925 magnification, note the presence of a large fibrin (red)/collagen(blue) scar. (P) IHC of the same heart at

926 30dpa labelled with an anti- $\alpha$ sarcomeric actin antibody (green), note the large area beneath the white

927 dashed line which is unlabeled indicating an absence of cardiomyocytes. 
bioRxiv preprint doi: https://doi.org/10.1101/2021.10.25.465720; this version posted October 26, 2021. The copyright holder for this preprint (which was not certified by peer review) is the author/funder. All rights reserved. No reuse allowed without permission.

Figure 4

A

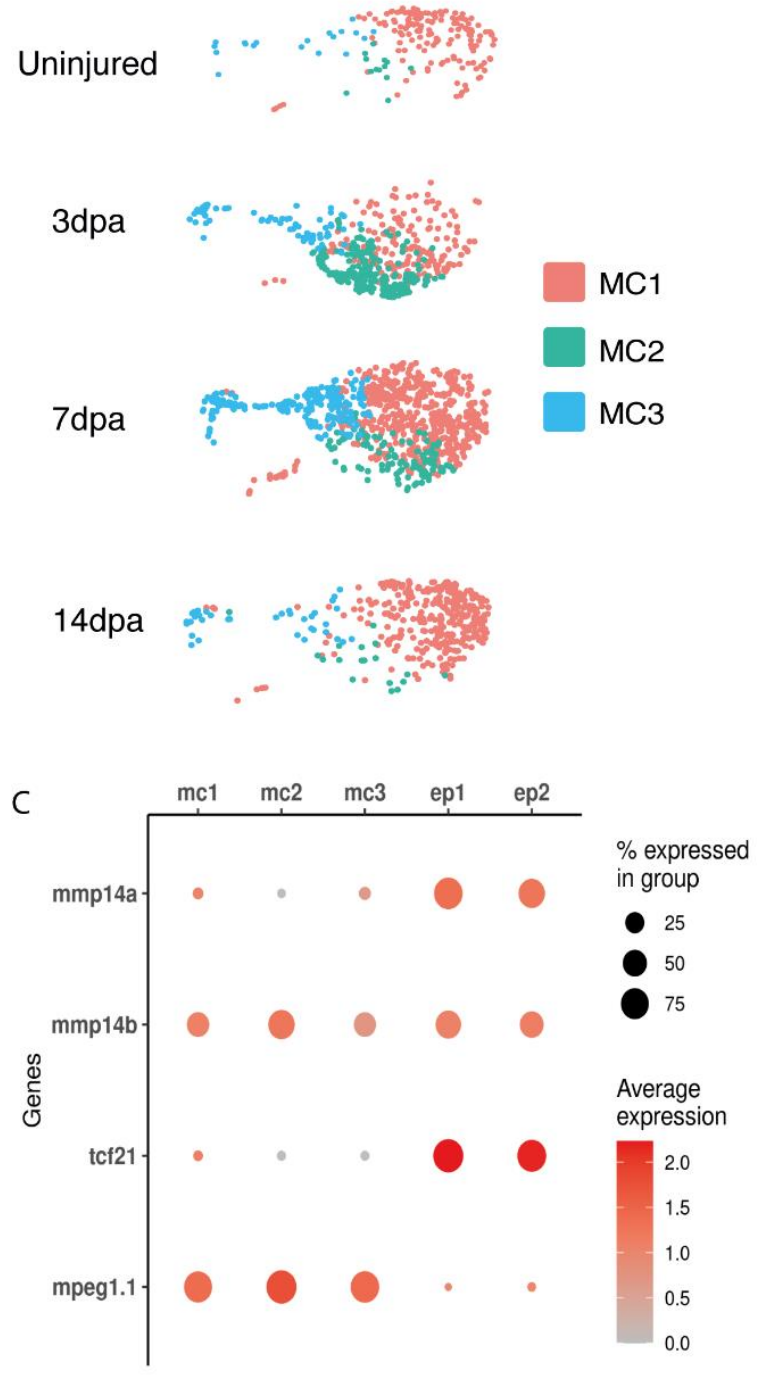

B

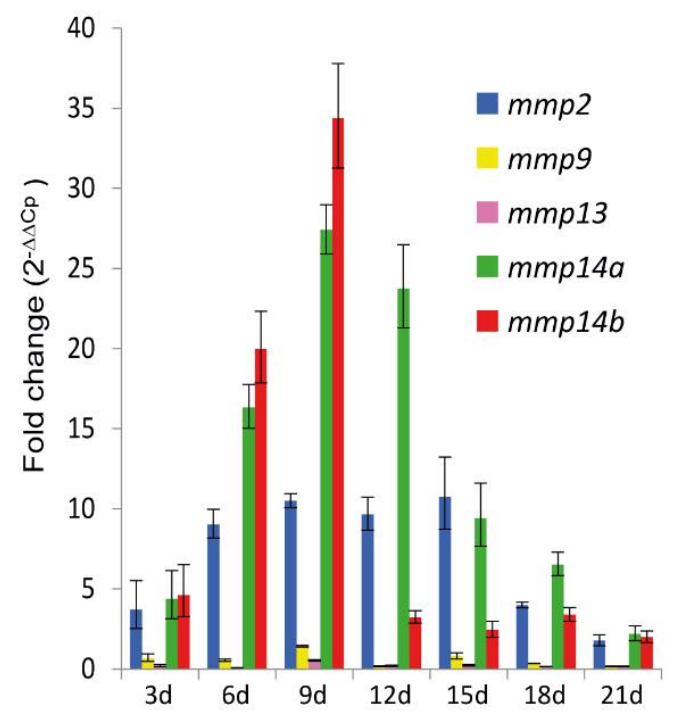

D

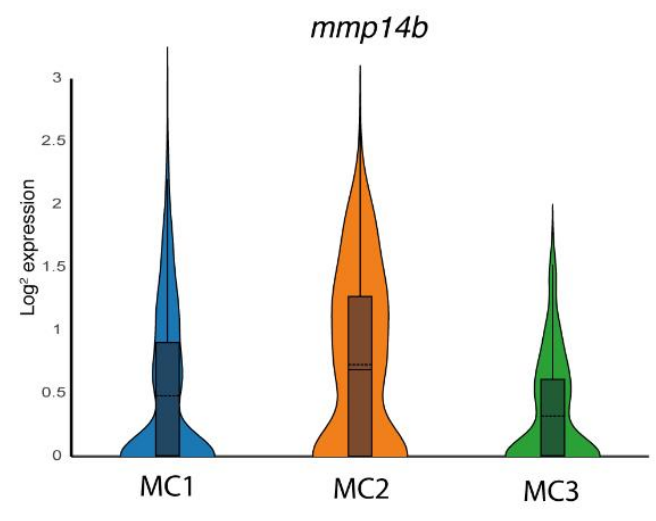

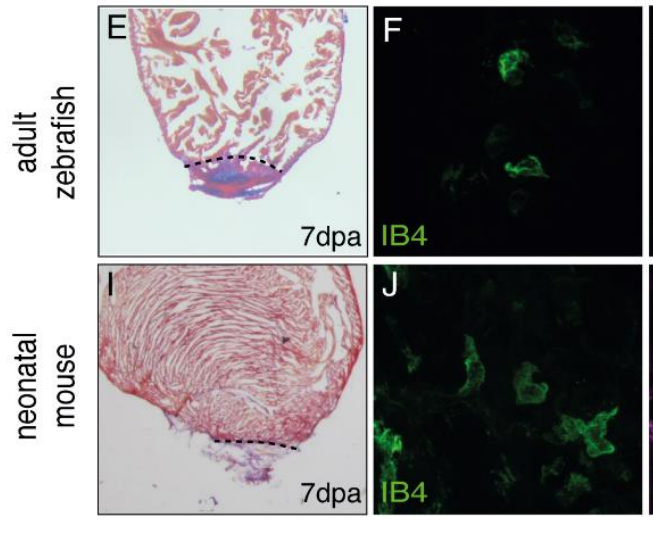
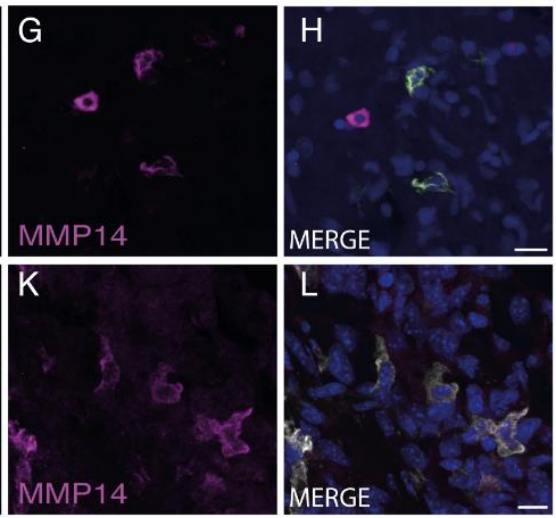
929 Figure 4. MMP14 is expressed by macrophages during cardiac regeneration. (A) UMAP plots of the

930 macrophage population at different stages of regeneration (uninjured, 3dpa, 7dpa and $14 \mathrm{dpa}$ ). The coloured

931 boxes indicate the different macrophage clusters (MC1-3). (B) RT-QPCR analysis of mmp expression

932 during cardiac regeneration. (C) A DotPlot showing the relative expression levels of $m m p 14 a$ and $m m p 14 b$

933 in macrophages (MC1-3) and fibroblasts/epicardium (FB1,2). $t c f 21$ was used to identify the fibroblast

934 population and mpeg1.1 the macrophage population. (D) Violin plot comparing the expression of $m m p 14 b$

935 in either the MC1, MC2 or MC3 macrophage clusters. (E) AFOG staining of a wildtype zebrafish heart at

936 7dpa, black dashed line indicates the plane of amputation. Note the presence of a large fibrin

937 (red)/collagen(blue) scar. (F-H) IHC of a 7dpa zebrafish heart section for (F) IB4 (green, macrophages),

938 (G) Mmp14 (magenta) and a merged image of F and G (scale bar 10 $\mu \mathrm{m})(\mathbf{H})$. (I) AFOG staining of a

939 neonatal mouse heart section at 7dpa, black dashed line indicates the plane of amputation. Note the presence

940 of a large fibrin (red)/collagen(blue) scar. (J-L). IHC on a neonatal mouse heart section at 7 dpa (J) IB4

941 (green, macrophages), (K) MMP14 (magenta) and a merged image of J and K (L). 
Figure 5
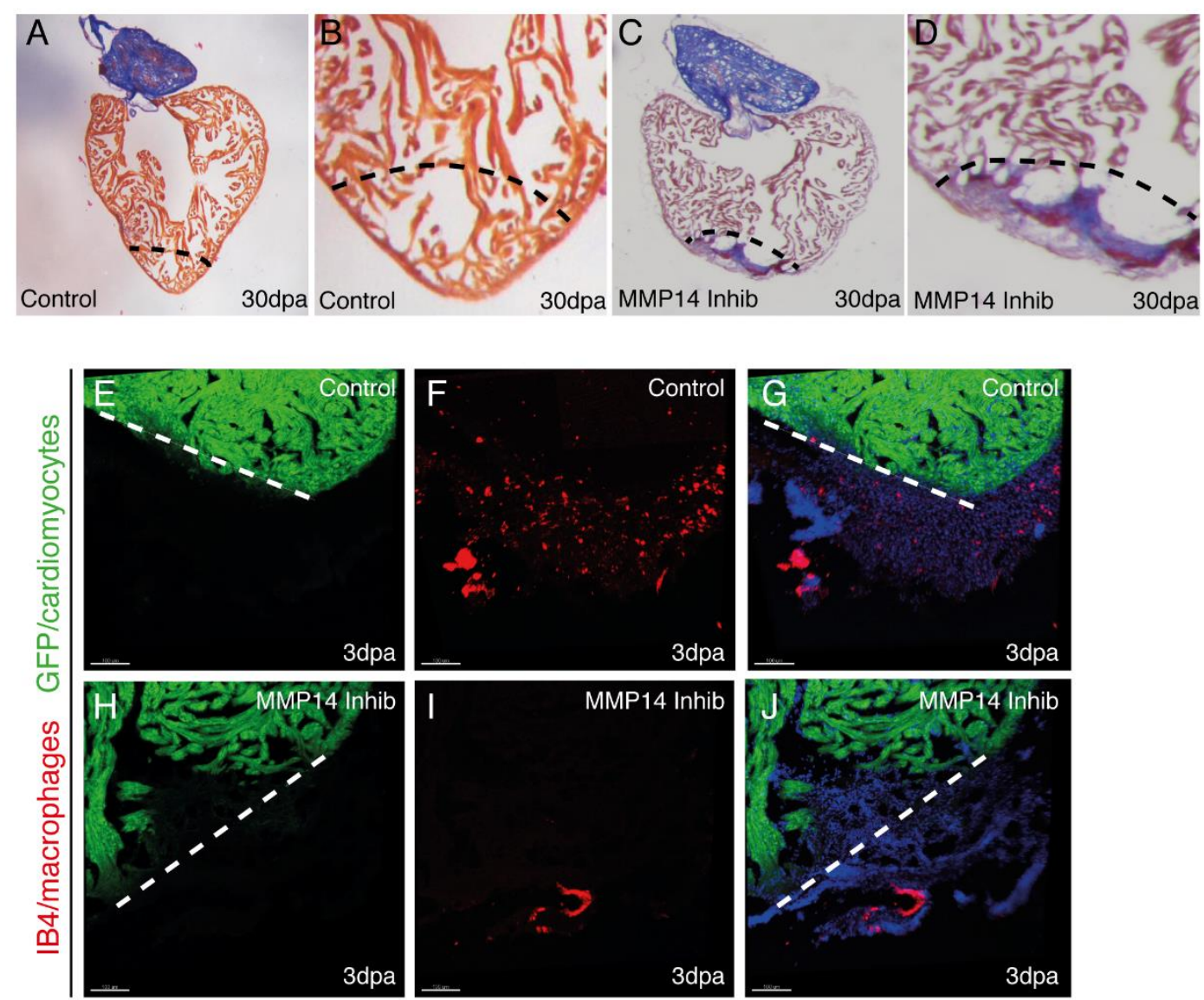

K

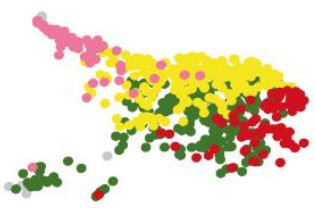

3dpa control

$3 d p a+$ NSC40520

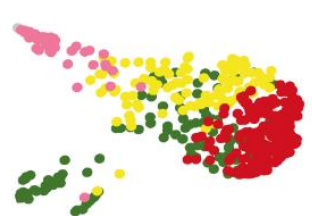

M

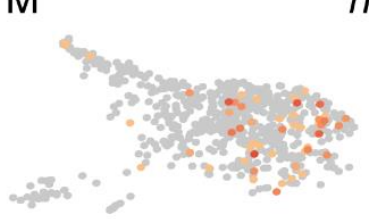

hbegfb

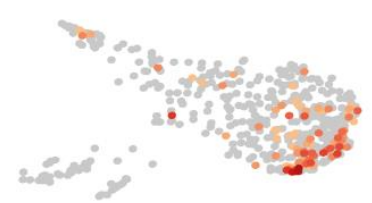

L

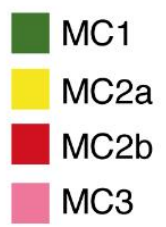

$m m p 14 b$

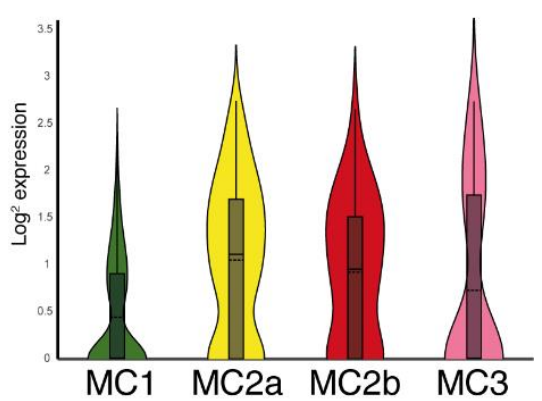


944 staining of a control zebrafish heart at 30dpa, black dashed line indicates the plane of amputation (scale bar

$945200 \mu \mathrm{m})$. (C,D) AFOG staining of a MMP14 inhibitor treated zebrafish heart at 30dpa, black dashed line

946 indicates the plane of amputation (scale bar $200 \mu \mathrm{m}$ ). Note the presence of a large fibrin (red)/collagen(blue)

947 scar. (E-G) IHC on a 3dpa control zebrafish heart section for (E) cardiomyocytes (GFP, green) (scale bar

$948100 \mu \mathrm{m}),(\mathbf{F})$ macrophages (IB4, red) and a merged image of $\mathrm{E}$ and $\mathrm{F}(\mathbf{G})$. Note the presence of numerous

949 macrophages in (F). (H-J) IHC on a 3dpa MMP14 inhibitor treated zebrafish heart section for (H)

950 cardiomyocytes (GFP, green) (scale bar 100 $\mu \mathrm{m}$ ), (I) macrophages (IB4, red) and a merged image of $\mathrm{H}$ and

951 I (J). Note the absence of macrophages in (I). (K) UMAP plots of the macrophage population in control

952 and MMP14 inhibitor treated zebrafish hearts at 3dpa. The coloured boxes indicate the different

953 macrophage clusters (MC1-3). (L) Violin plot comparing the expression of mmp14b in the MC1, MC2a,

$954 \mathrm{MC} 2 \mathrm{~b}$ and MC3 macrophage clusters. (M) UMAP plots depicting the relative expression of hbefgb in

955 control and MMP14 inhibitor treated macrophages. Note the increased expression of hbefg $b$ in the MMP14

956 inhibitor treated recruited macrophage cluster (MC2b). 
bioRxiv preprint doi: https://doi.org/10.1101/2021.10.25.465720; this version posted October 26, 2021. The copyright holder for this preprint (which was not certified by peer review) is the author/funder. All rights reserved. No reuse allowed without permission.

\section{Supplemental Figure 1}

A

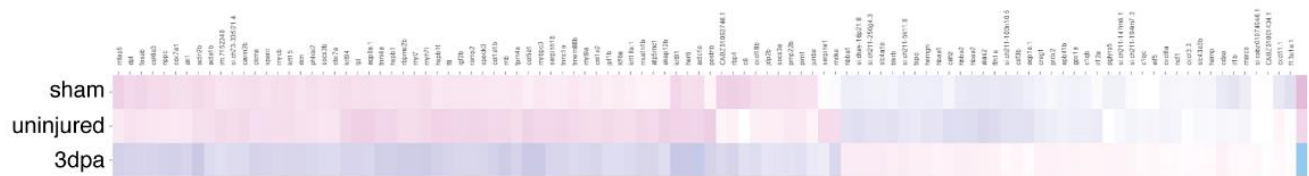

B

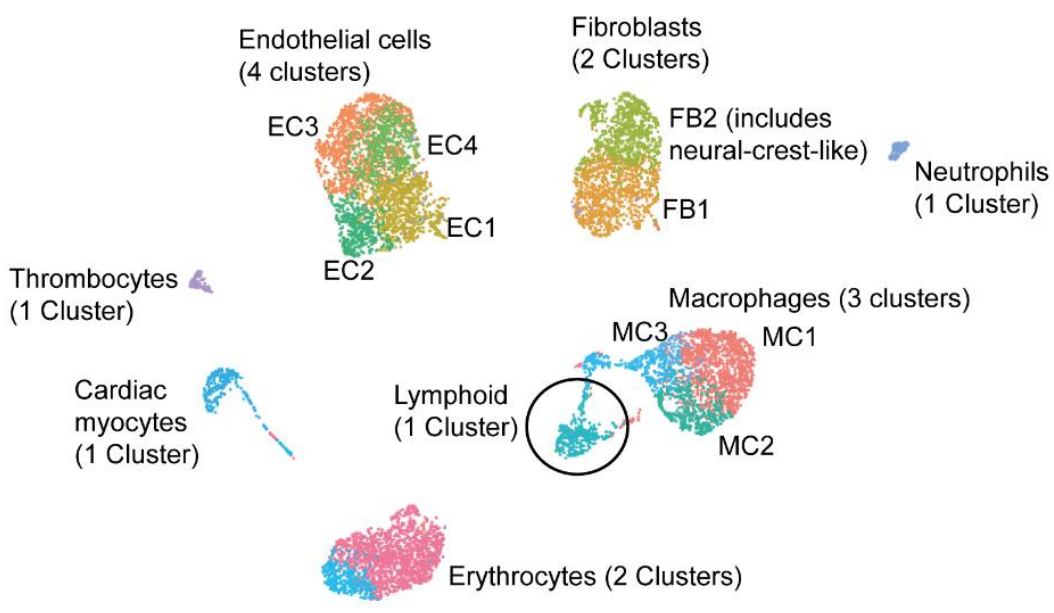

C
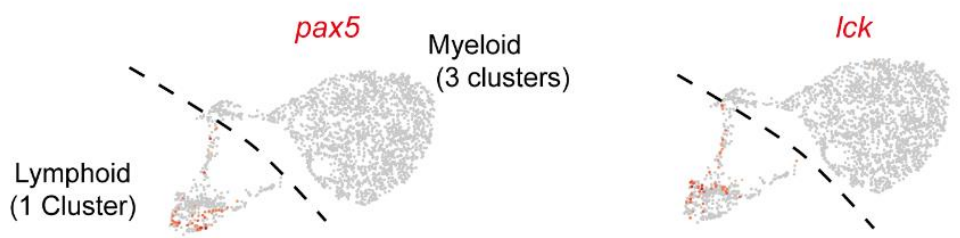

D
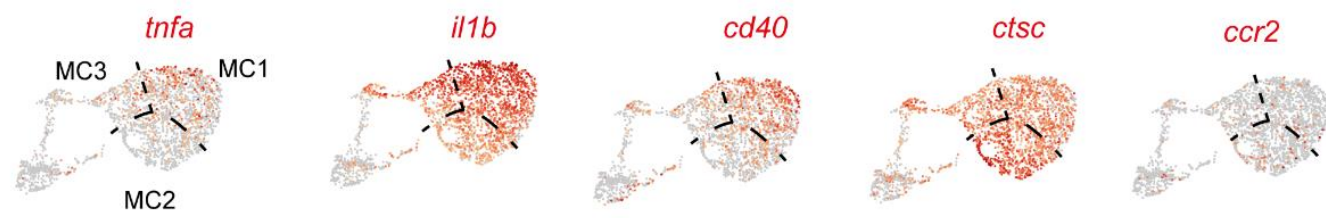

E
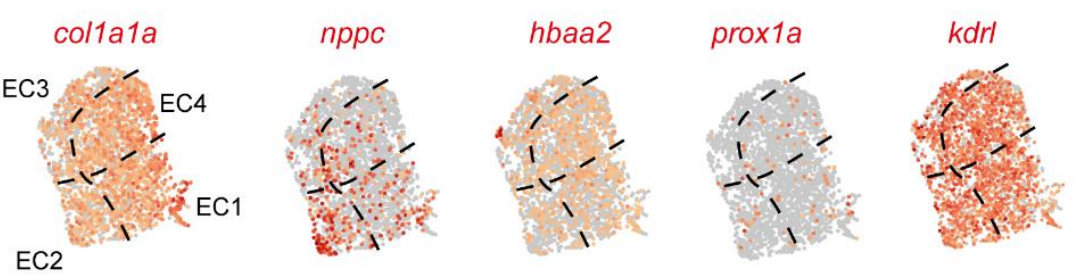

$\mathrm{F}$
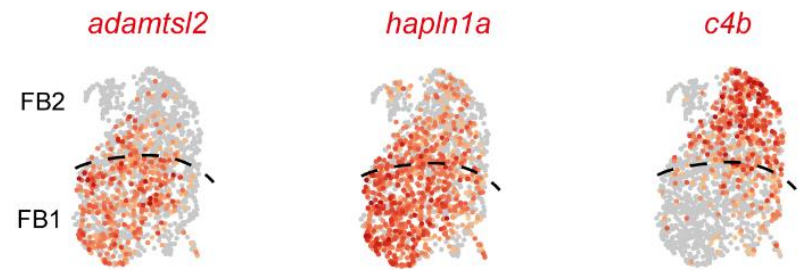

aldh1a2 
958 Supplemental Figure.1. Single cell RNAseq analysis of regenerating zebrafish hearts. (A) Heat map

959 depicting differentially expressed genes among sham operated, uninjured and 3dpa samples. (B) UMAP

960 plot highlighting the different clusters within in each population of cells identified in regenerating zebrafish

961 hearts. In particular, 4 clusters were identified within the endothelial population of cells (EC1-4), 2 clusters

962 were identified within the epicardium/fibroblast population of cells (FB1,2) and 3 clusters were identified

963 within the myeloid/macrophage population of cells (MC1-3). (C-F) UMAP plots depicting the relative

964 expression of particular genes within the different sub clusters. (C) Lymphoid cells (pax5 and lck). (D)

965 Macrophages (tnfa, illb, cd40, ctsc, ccr2). (E) Endothelium (collala, nppc, hbaa2, proxla, kdrl). (F)

966 Fibroblasts (adamtsl1, hapln1a, c4b, aldhla2). 


\section{Supplemental Figure 2}

A

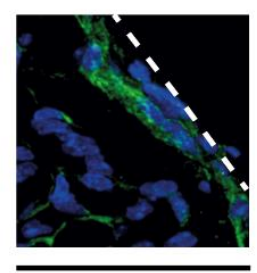

DAPI Rspo1

C

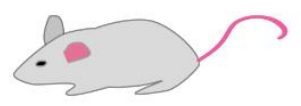

Adult mouse myocardial infarction

Re-analysed data from Farbehi et al.
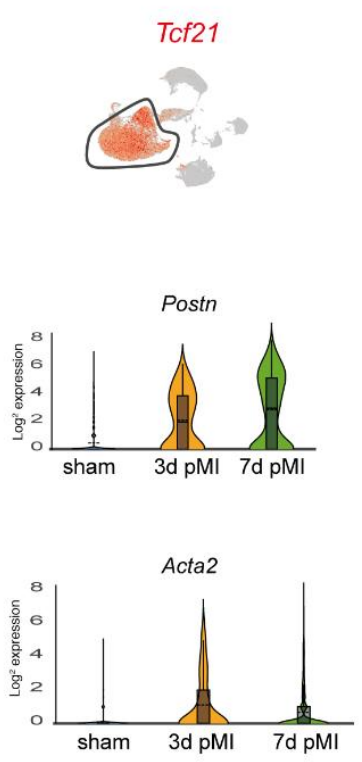

B Re-analysed data from Wang et al.
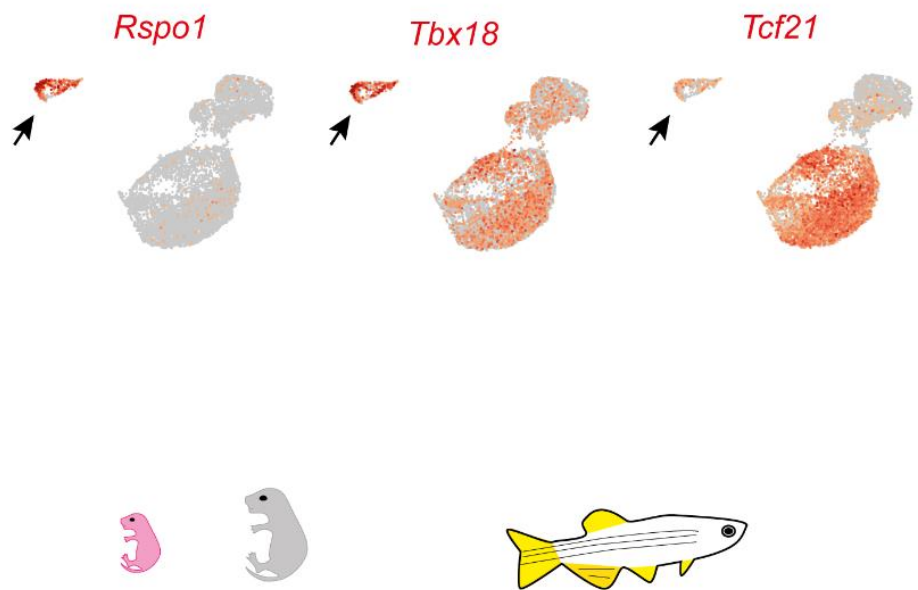

Neonatal mouse heart myocardial infarction Re-analysed data from Wang et al.

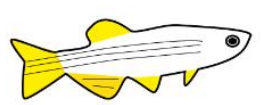

Adult zebrafish apical ressection

Tcf21

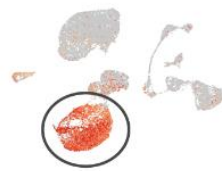

tcf21

0
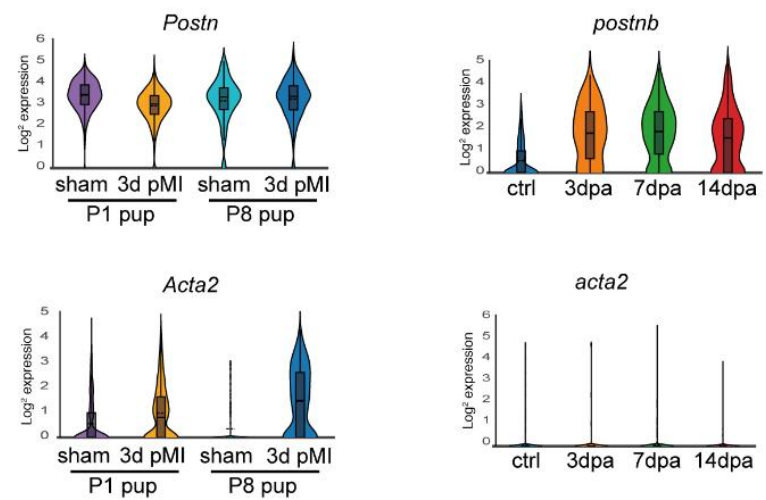

Supplemental Figure 2. Mesenchymal lineages and cardiac fibroblast activation in the regenerating

969 zebrafish heart. (A) IHC image of 7dpa neonatal mouse heart labelled with RSPO1 antibody (green), white

970 dashed line indicates the outer edge of the epicardium. (B) UMAP plots from re-analysed neonatal mouse

971 scRNA-seq data (Wang et al.) depicting the co-expression of Rspol with Tbx18 and Tcf21. Note that

972 although Rspol is co-expressed with both genes in a smaller cluster (black arrow) there is relatively little 
973 expression within the main $T c f 21^{+}$fibroblast cluster. (C) Re-analysis of scRNA-seq data from adult

974 (Farbehi et al.) and neonatal mice (Wang et al.) after myocardial infarction compared with adult zebrafish

975 after apical resection. Adult mouse data is from 3 and 7 days post myocardial infarction (MI). Neonatal is

976 from postnatal day 1 and postnatal day 8 pups, with sham and 3 days post MI samples. UMAP plots

977 highlight the fibroblast population $(T c f 21)$ in each dataset. Violin plots represent the expression of Periostin

978 and Acta2 at different time points after injury in each dataset. Note- Periostin is expressed in uninjured

979 neonatal mouse hearts, Acta2 is expressed by fibroblasts in both adult and neonatal mice after injury but

980 not by adult zebrafish fibroblasts. 


\section{Supplemental Figure 3}

A

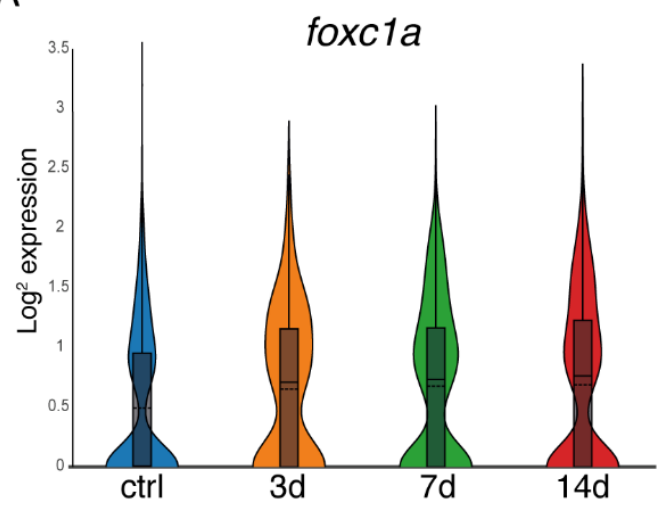

C

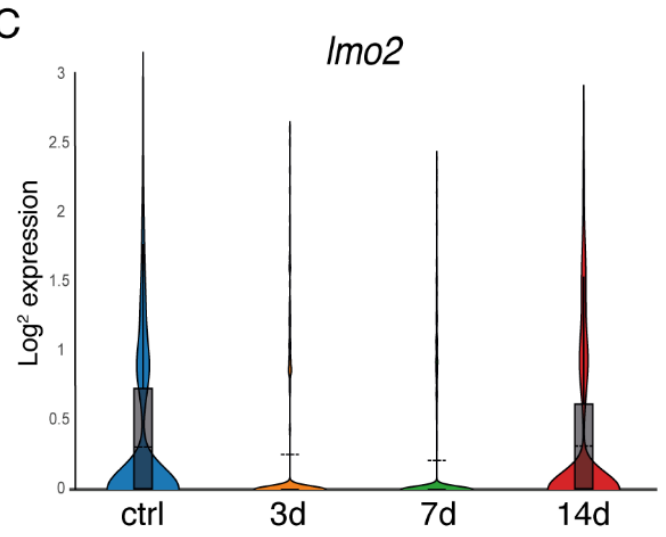

B foxc1b

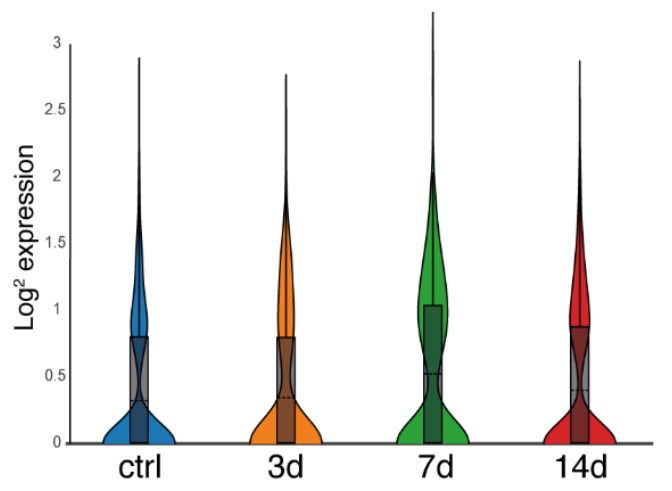

Supplemental Figure 3. Tal1 is a regulator of the endothelial regenerative response. (A-C) Violin plots 
bioRxiv preprint doi: https://doi.org/10.1101/2021.10.25.465720; this version posted October 26, 2021. The copyright holder for this preprint (which was not certified by peer review) is the author/funder. All rights reserved. No reuse allowed without permission.

Supplemental Figure 4
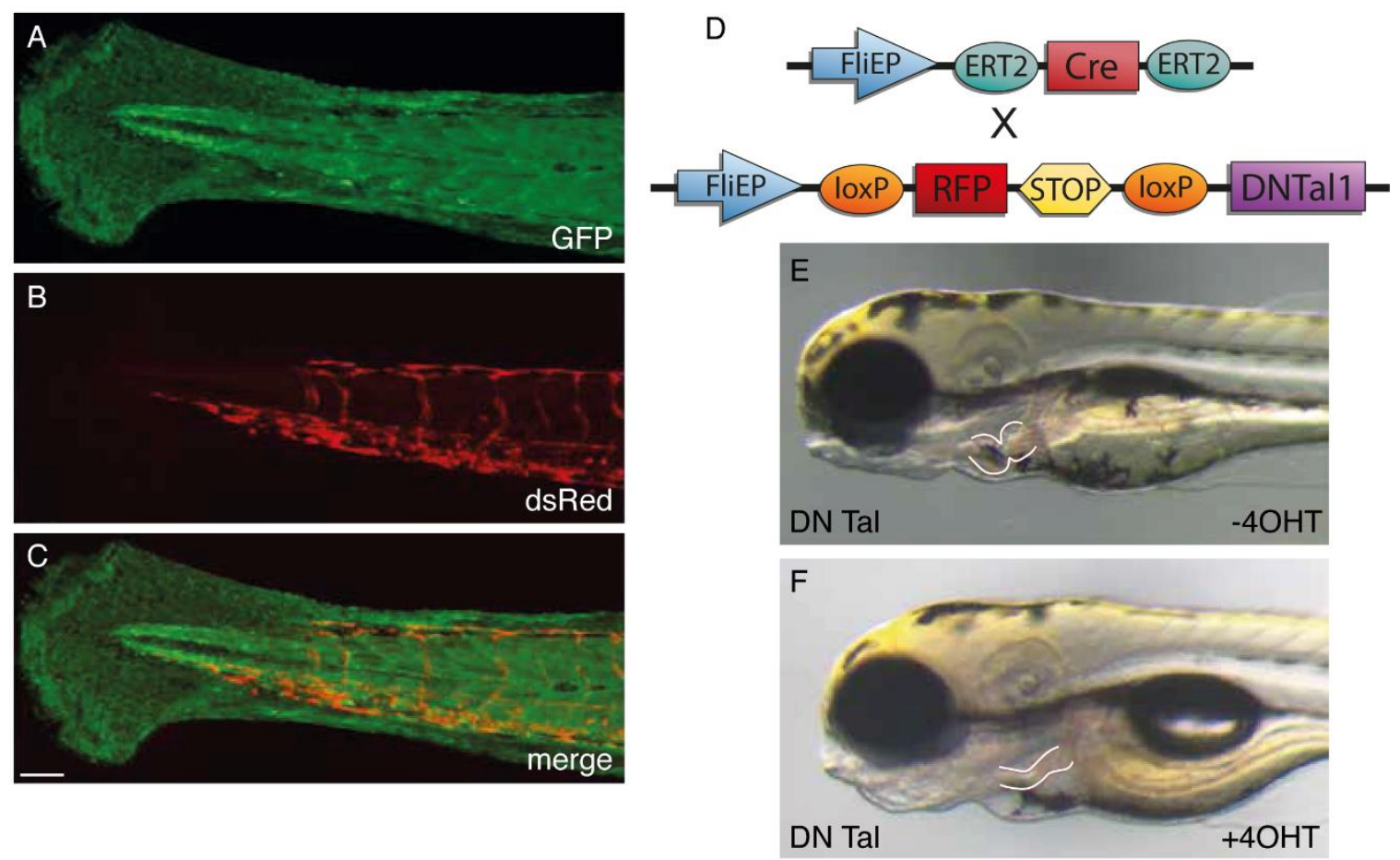

G

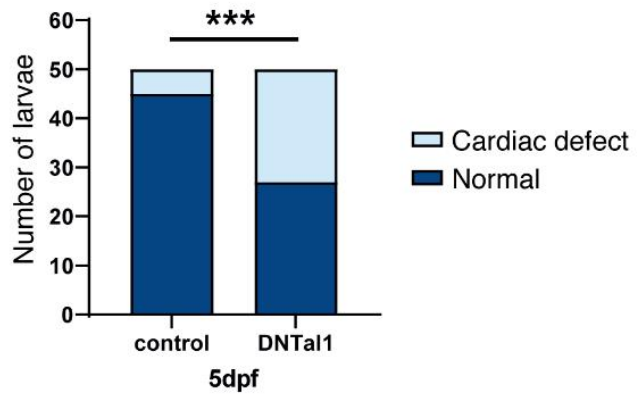

$\mathrm{H}$

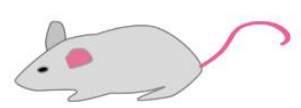

Adult mouse myocardial infarction

Re-analysed data from Farbehi et al.

Tal1

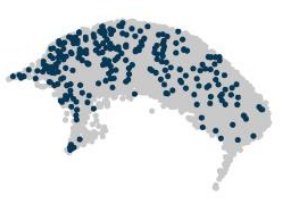

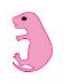

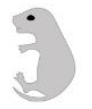

Neonatal mouse heart myocardial infarction Re-analysed data from Wang et al.

Tal1

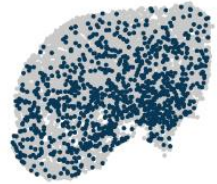

tal1

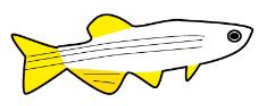

Adult zebrafish apical ressection

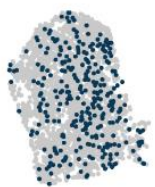


986 Supplemental Figure 4. Tal1 is a regulator of the endothelial regenerative response. (A-C) IHC images

987 of a Tg(eab2:[EGFP-T-mCherry])vu295 / Tg(fliEP:Ert2CreErt2) 5dpf larvae treated with tamoxifen. (A)

988 GFP is ubiquitously expressed. (B) DsRed is expressed in endothelial cells following Cre mediated

989 recombination. (C) Merged image of A and B. Note the vasculature is labelled with dsRed (B) indicating

990 that Ert2CreErt2 in Tg(fliEP:Ert2CreErt2) zebrafish is restricted to endothelial cells. (D) Diagram

991 indicating the 2 constructs used to generate the Tg(fliEP:Ert2CreErt2;fliEP:loxRFPlox:DNtal) transgenic

992 zebrafish line. (E,F) Tg(fliEP:Ert2CreErt2;fliEP:loxRFPlox:DNtal) transgenic zebrafish larvae either

993 untreated -4OHT (E) or tamoxifen treated +4OHT $(\mathbf{F})$. The developing heart has been highlighted with

994 white lines, note that the heart in the tamoxifen treated larvae has failed to develop normally. (G) Graph

995 depicting the number of larvae which develop a cardiac defect in either untreated (control) or tamoxifen

996 treated (DN Tal1) groups. Fisher's exact test, $\mathrm{n}=50$ larvae for each condition $(* * *: \mathrm{p}<0.001)$. (H) Re-

997 analysis of scRNA-seq data from post myocardial infarction adult mice (Farbehi et al.) and neonatal mice

998 (Wang et al.) compared with adult zebrafish after apical resection. UMAP plots indicating the expression

999 of Tall (dark blue dots) within the endothelial population of cells in each dataset. 


\section{Supplemental Figure 5}

A

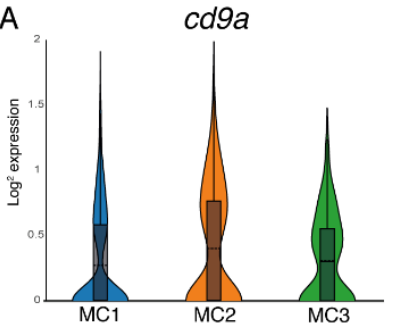

C

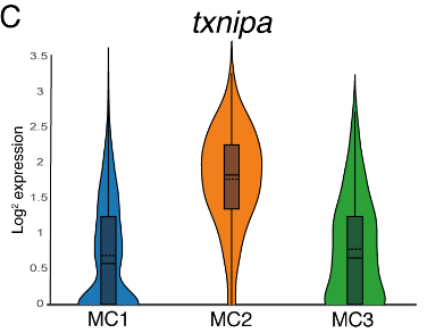

E

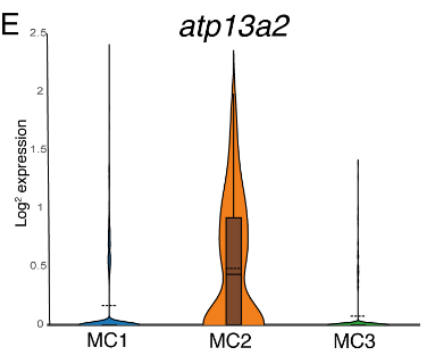

G

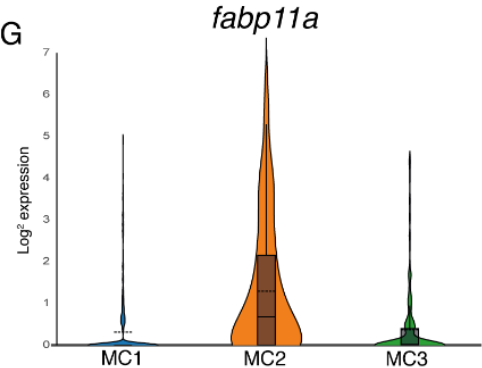

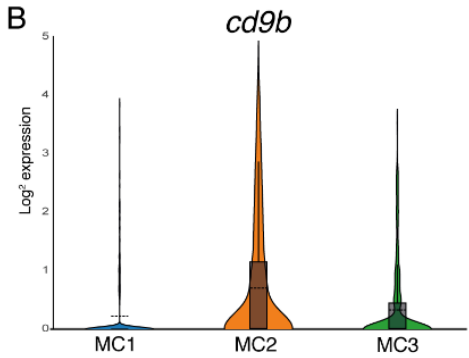

D
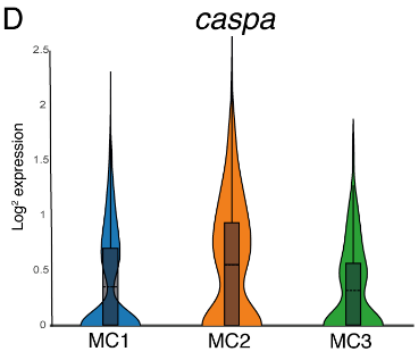

F
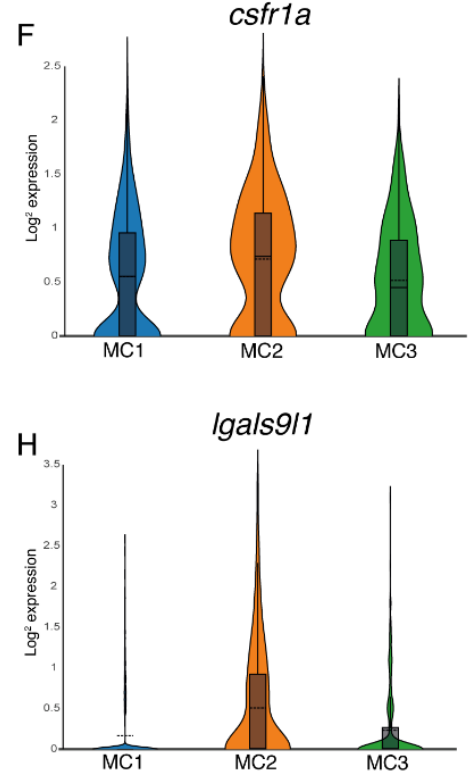

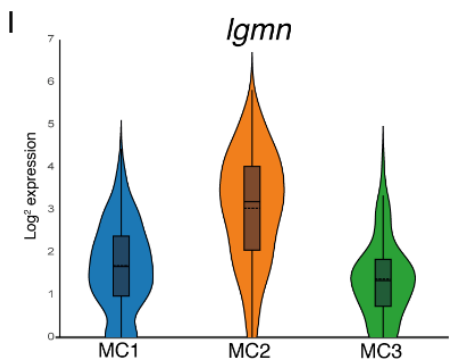


1001 Supplemental Figure 5. Genes enriched in the recruited macrophage population. (A-I) Violin plots

1002 comparing the expression of different genes within the macrophage subpopulations (MC1-resident

1003 macrophages, MC2-recruited macrophages and MC3- proliferating macrophages), $c d 9 a(\mathbf{A}), c d 9 b(\mathbf{B})$,

1004 txnipa $(\mathbf{C})$, caspa (D), atp13a2 (E), csfrla (F), fabpl la (G), lgals9l1 (H) and lgmn (I).

1005

Supplemental Figure 6
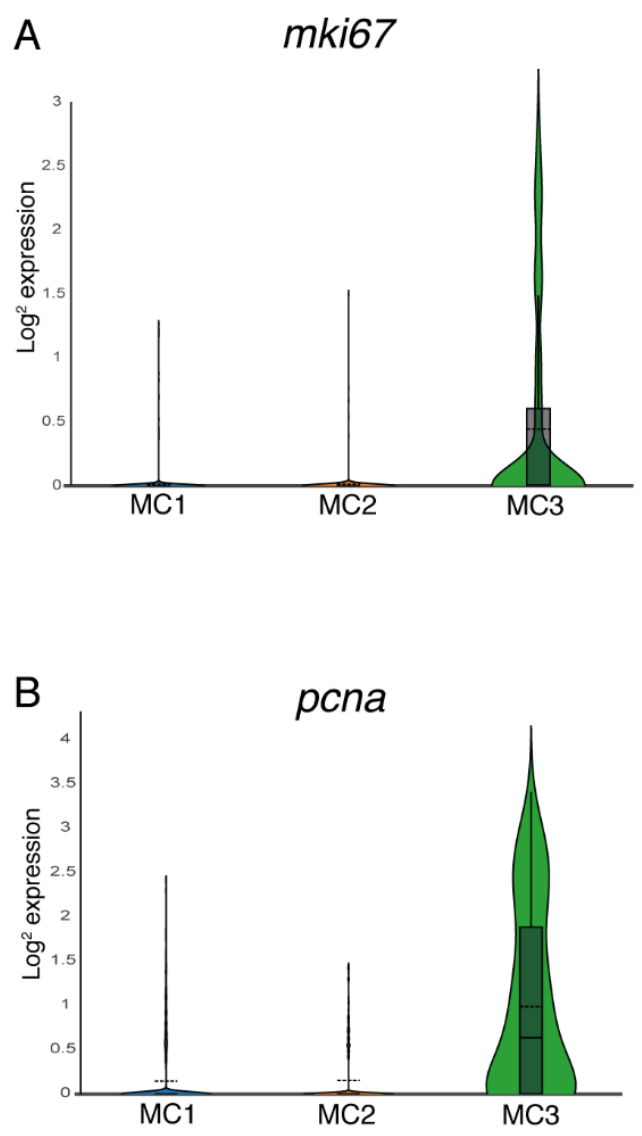
1007 Supplemental Figure 6. Genes enriched in the proliferating macrophage population. (A,B) Violin

1008 plots comparing the expression of different genes within the macrophage subpopulations (MC1-

1009 proliferating macrophages, MC2-recruited macrophages and MC3-resident macrophages), mki67 (A) and 1010 pcna $(\mathbf{B})$.

\section{Supplemental Figure 7}
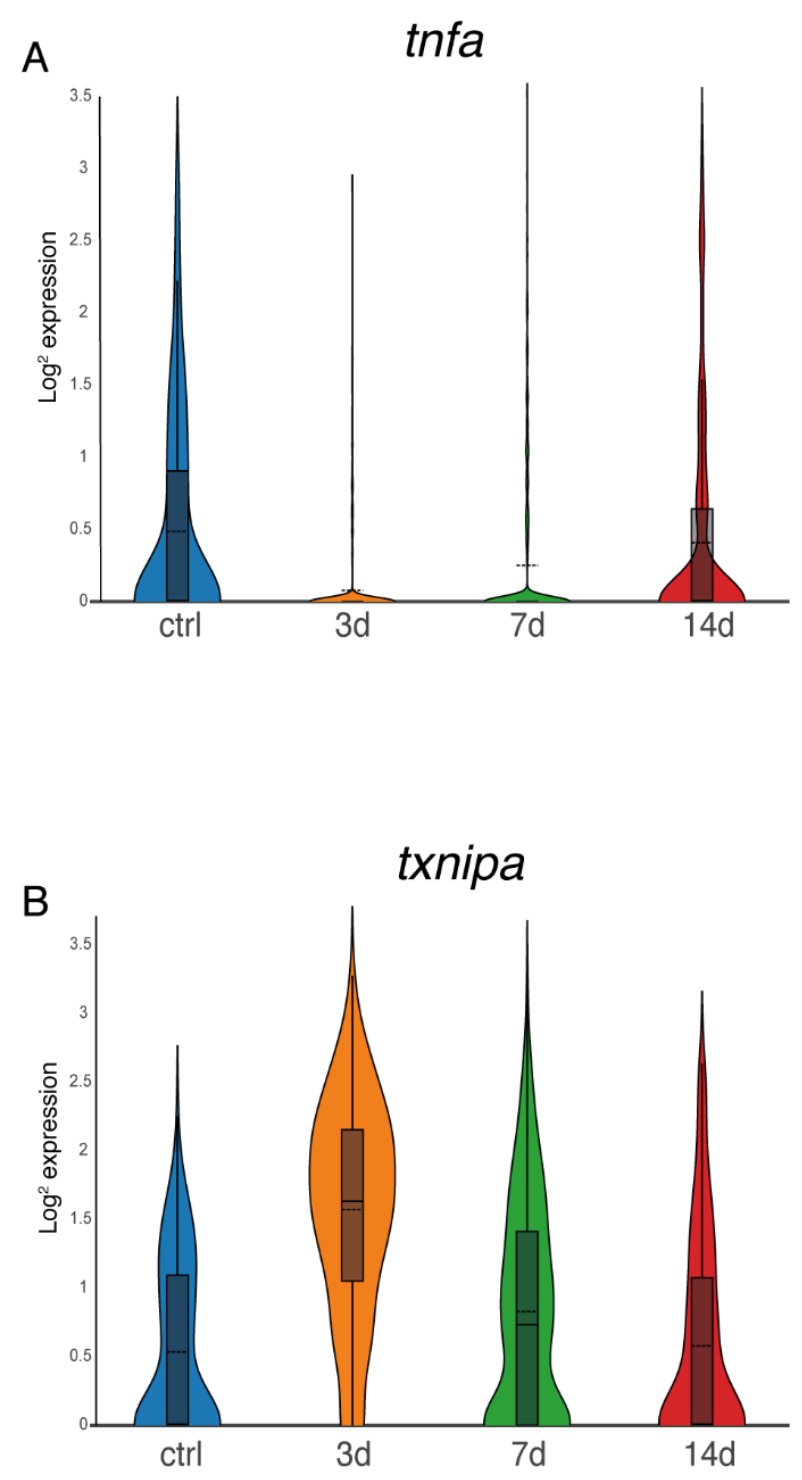
bioRxiv preprint doi: https://doi.org/10.1101/2021.10.25.465720; this version posted October 26, 2021. The copyright holder for this preprint (which was not certified by peer review) is the author/funder. All rights reserved. No reuse allowed without permission.

1012 Supplemental Figure 7. Dynamic expression of inflammatory genes in macrophages at different time

1013 points during cardiac regeneration. (A,B) Violin plots comparing the expression of different genes by

1014 macrophages at different time points during cardiac regeneration (uninjured (ctrl), 3dpa, 7dpa and 14dpa),

1015 tnfa (A), and txnipa (B). 


\section{Supplemental Figure 8}

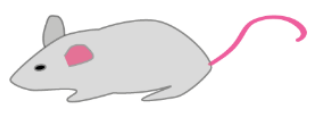

$$
\begin{aligned}
& \text { Adult mouse } \\
& \text { myocardial infarction }
\end{aligned}
$$

Re-analysed data from Farbehi et al.

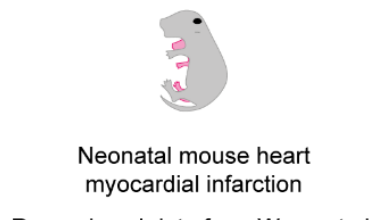

Re-analysed data from Wang et al.

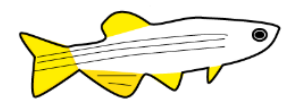

Adult zebrafish apical ressection
Ccr2

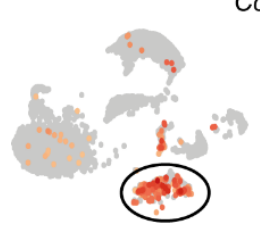

Uninjured

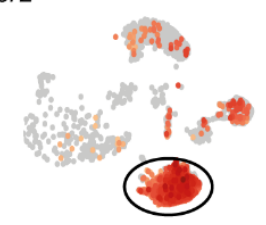

3d post injury
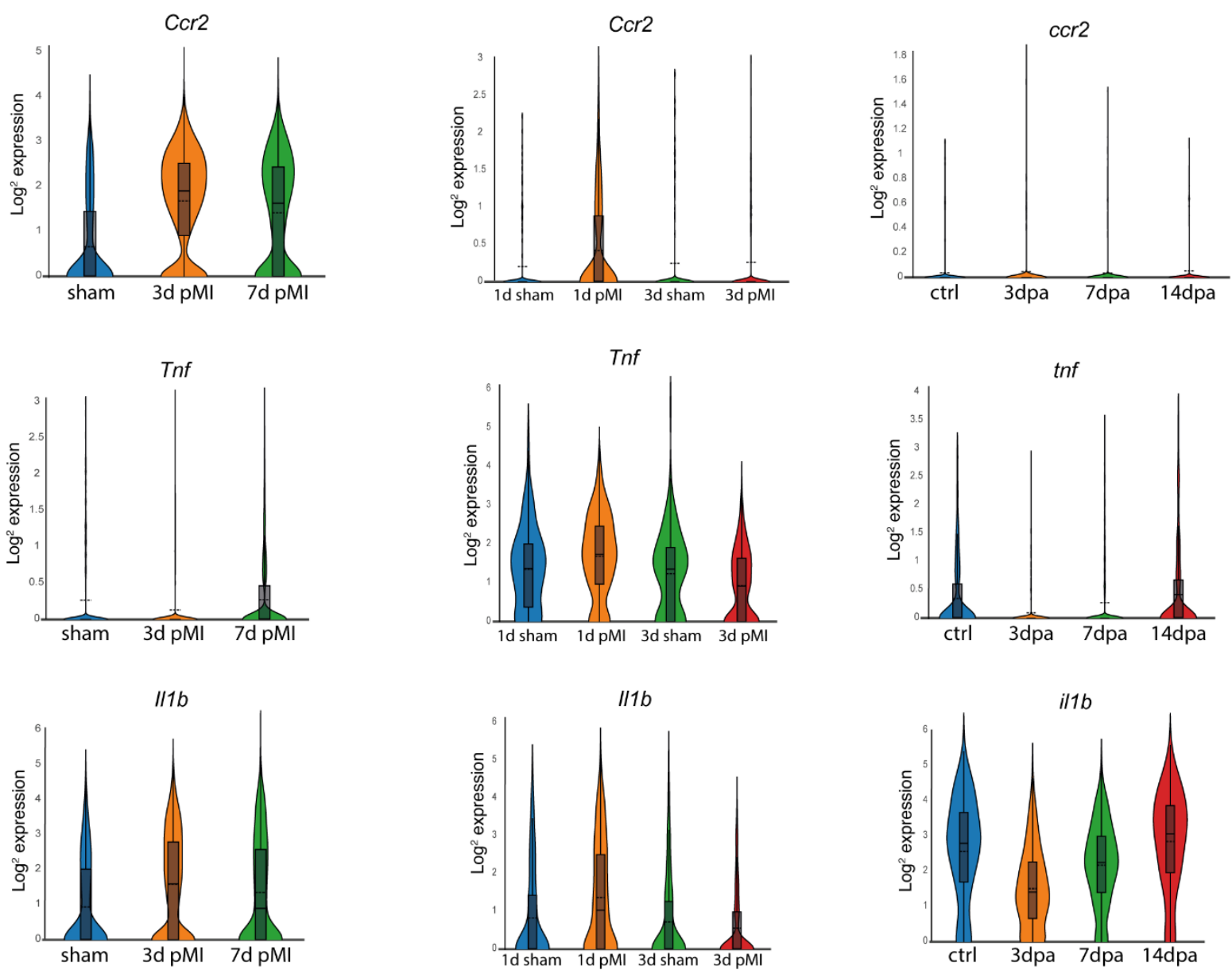

Ccr2

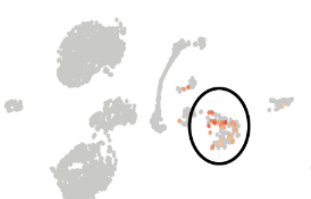

Uninjured

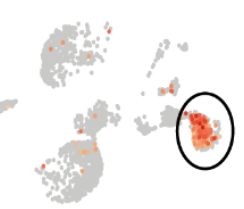

1d post injury ccr2

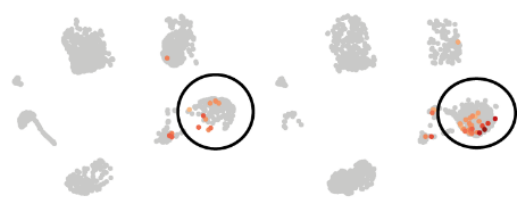

Uninjured

3d post injury
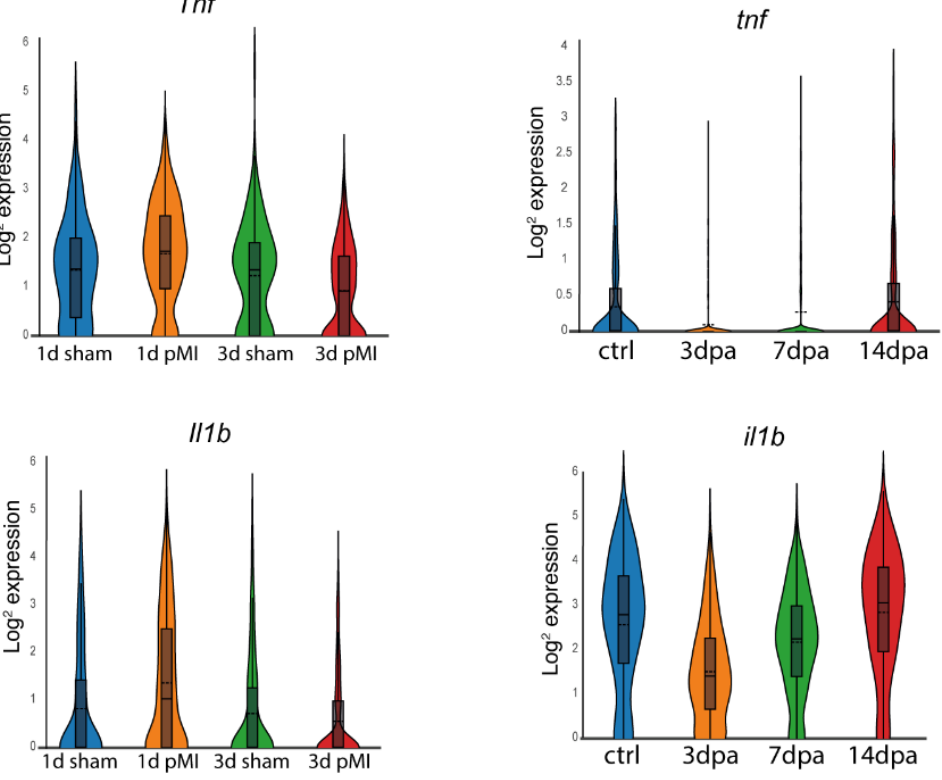
1017 Supplemental Figure 8. Comparison of recruited macrophages between species. Adult mouse

1018 myocardial infarction. Re-analysed UMAP plots depicting the expression of $C c r 2$ in uninjured sham

1019 conditions vs 3 days post injury. Black circles indicate the macrophage population. Violin plots showing

1020 the expression of $C c r 2$, Tnfa and $I l l b$ at different time points after injury (sham, 3 days post myocardial

1021 infarction (d pMI) and 7d pMI) in macrophages. Neonatal mouse myocardial infarction. Re-analysed

1022 UMAP plots depicting the expression of $C c r 2$ in uninjured sham conditions vs 1 day post injury. Black

1023 circles indicate the macrophage population. Violin plots showing the expression of $C c r 2, T n f a$ and $I l l b$ at

1024 different time points after injury (1 day sham, 1 day post myocardial infarction $(\mathrm{d}$ pMI) and $3 \mathrm{~d}$ sham, $3 \mathrm{~d}$

1025 pMI) in macrophages. Adult zebrafish apical resection. UMAP plots depicting the expression of Ccr2 in

1026 uninjured control conditions vs 3 days post injury. Black circles indicate the macrophage population. Violin

1027 plots showing the expression of $C c r 2$, Tnfa and $I l l b$ at different time points after injury (control, 3 days

1028 post amputation (dpa) and 7dpa and 14dpa) in macrophages. 


\section{Supplemental Figure 9}
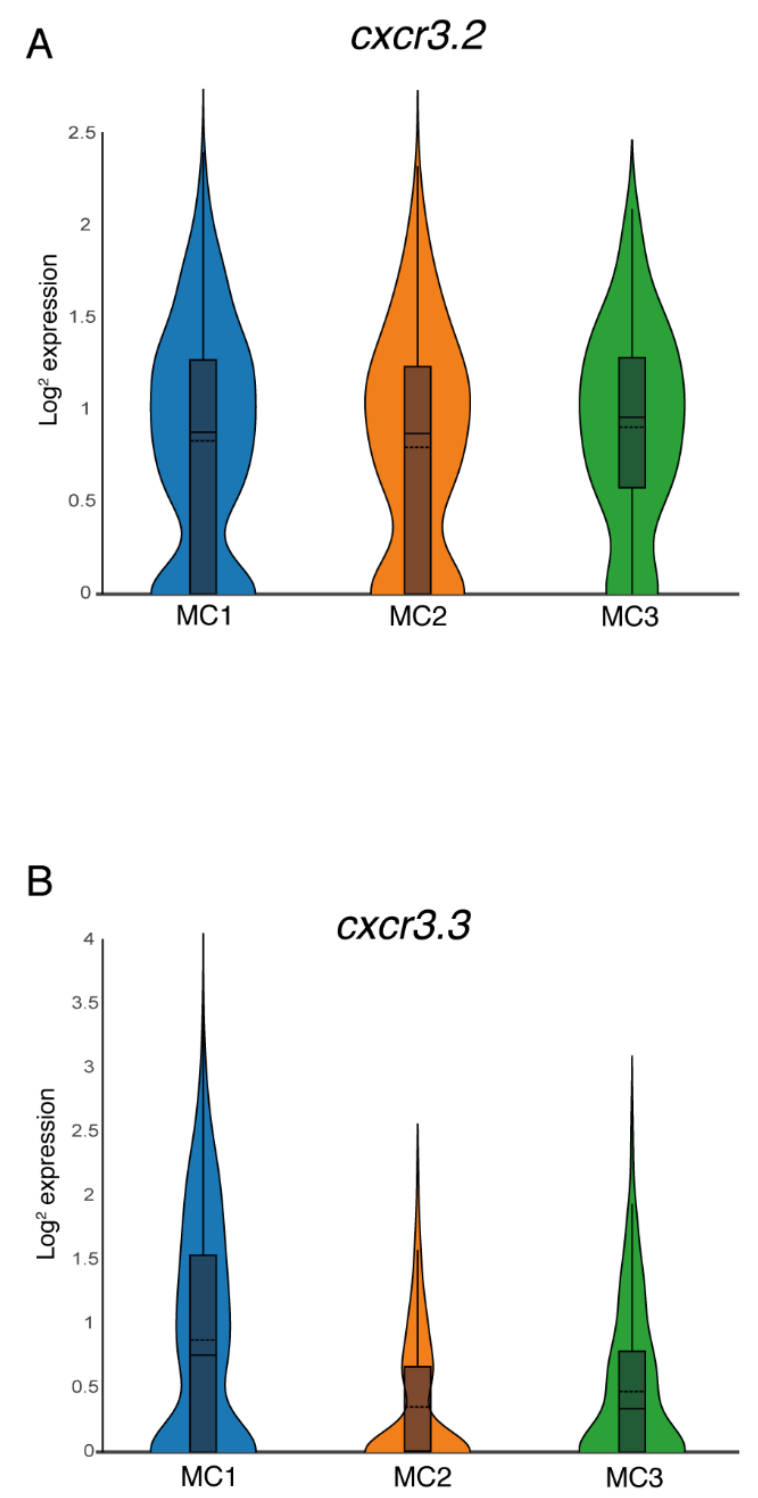

Supplemental Figure 9. The expression of $\operatorname{cxcr} 3.2$ and $\operatorname{cxcr} 3.3$ within the macrophage subpopulations.

1031 (A,B) Violin plots comparing the expression of $\operatorname{cxcr} 3.2$ and $\operatorname{cxcr} 3.3$ within the macrophage subpopulations

1032 (MC1- resident macrophages, MC2-recruited macrophages and MC3- proliferating macrophages), cxcr3.2

1033 (A) and $\operatorname{cxcr} 3.3$ (B). 


\section{Supplemental Figure 10}

A

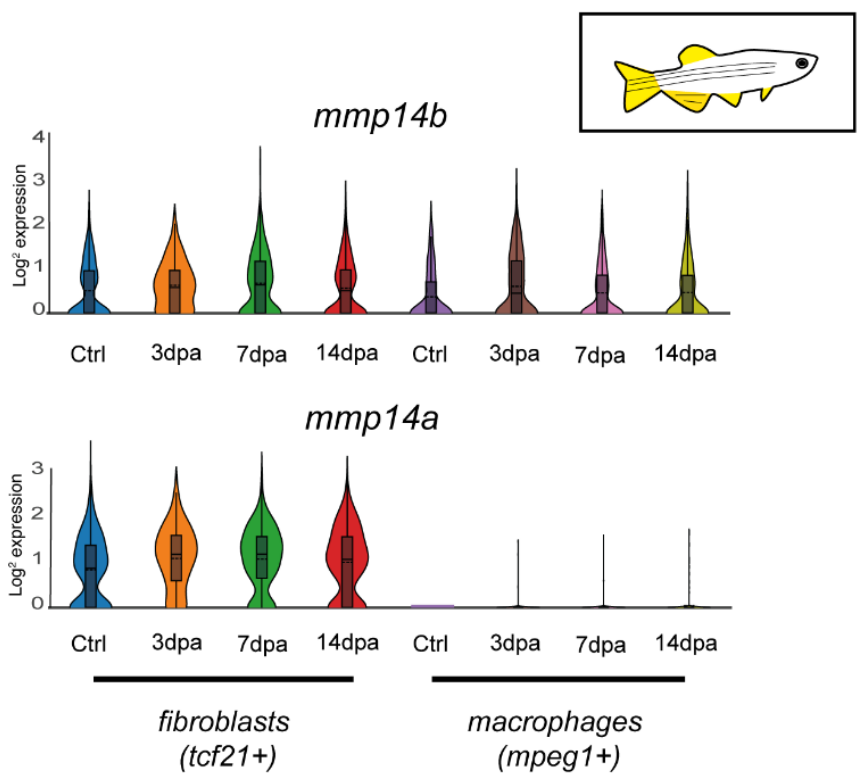

B Re-analysed data from Farbehi et al.
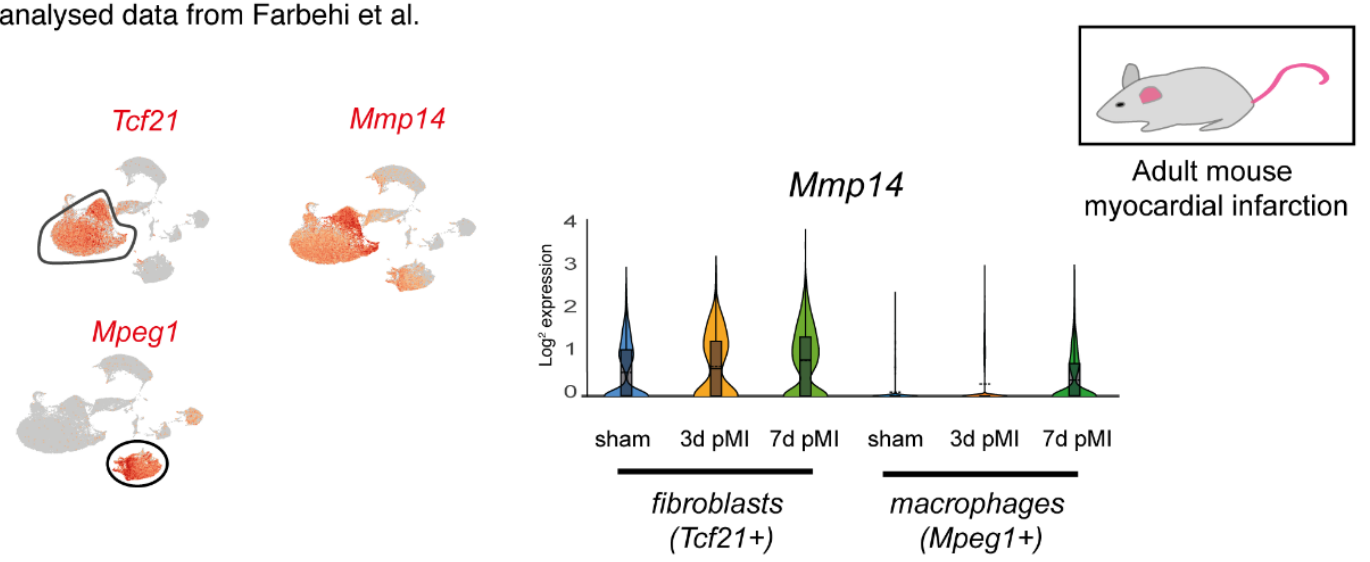

C Re-analysed data from Wang et al.

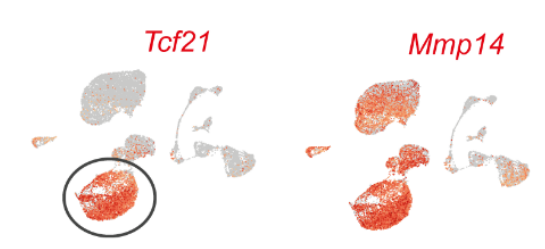

Mpeg1

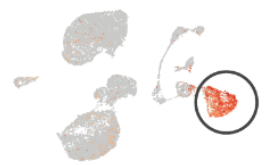

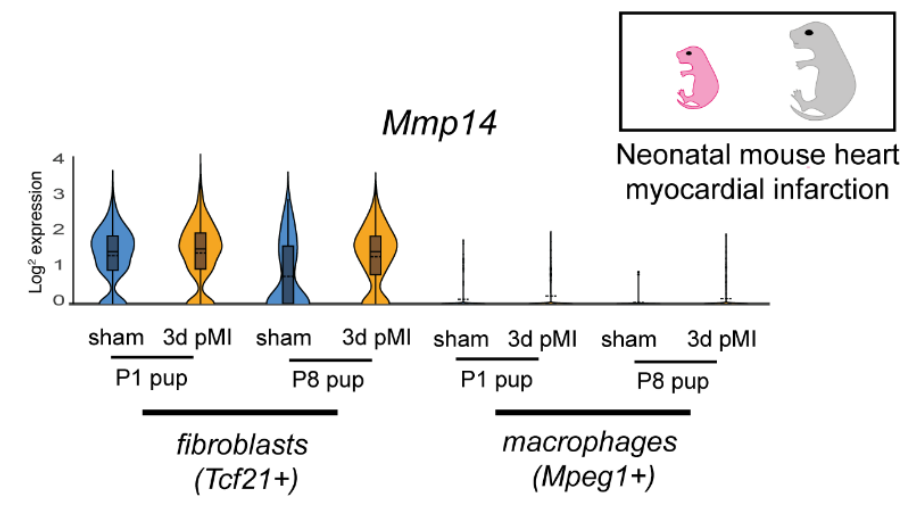


1035 Supplemental Figure 10. Comparison of Mmp14 expression in macrophages between species. (A)

1036 Adult zebrafish apical resection. UMAP plots depicting the expression of mmp14b and mmp14a in relation

1037 to $t c f 21$ expressing fibroblasts and mpeg1.1 expressing macrophages. Note mmpl4b is expressed by

1038 fibroblasts and macrophages while mmp $14 a$ is restricted to fibroblasts. Violin plots showing the expression

1039 of mmpl4b and mmpl4a at different time points during regeneration (uninjured (ctrl), 3dpa, 7dpa and

1040 14dpa) in fibroblasts and macrophages. (B) Adult mouse myocardial infarction. Re-analysed UMAP plots

1041 depicting the expression of Mmp14 in relation to Tcf21 expressing fibroblasts and Mpegl expressing

1042 macrophages. Violin plots showing the expression of Mmp14 at different time points after injury (sham, 3

1043 days post myocardial infarction (d pMI) and 7d pMI) in fibroblasts and macrophages. Note that Mmp14

1044 expression appears at 7d pMI in the macrophage population. (C) Neonatal mouse myocardial infarction.

1045 Re-analysed UMAP plots depicting the expression of Mmp14 in relation to Tcf21 expressing fibroblasts

1046 and Mpeg1 expressing macrophages. Violin plots showing the expression of Mmp14 at different time points

1047 after injury (sham, 3d pMI) by fibroblasts and macrophages in regenerating P1 pups and non-regenerating

1048 P8 pups. Note that Mmp14 expression is absent in the macrophage population. 
Supplemental Figure 11
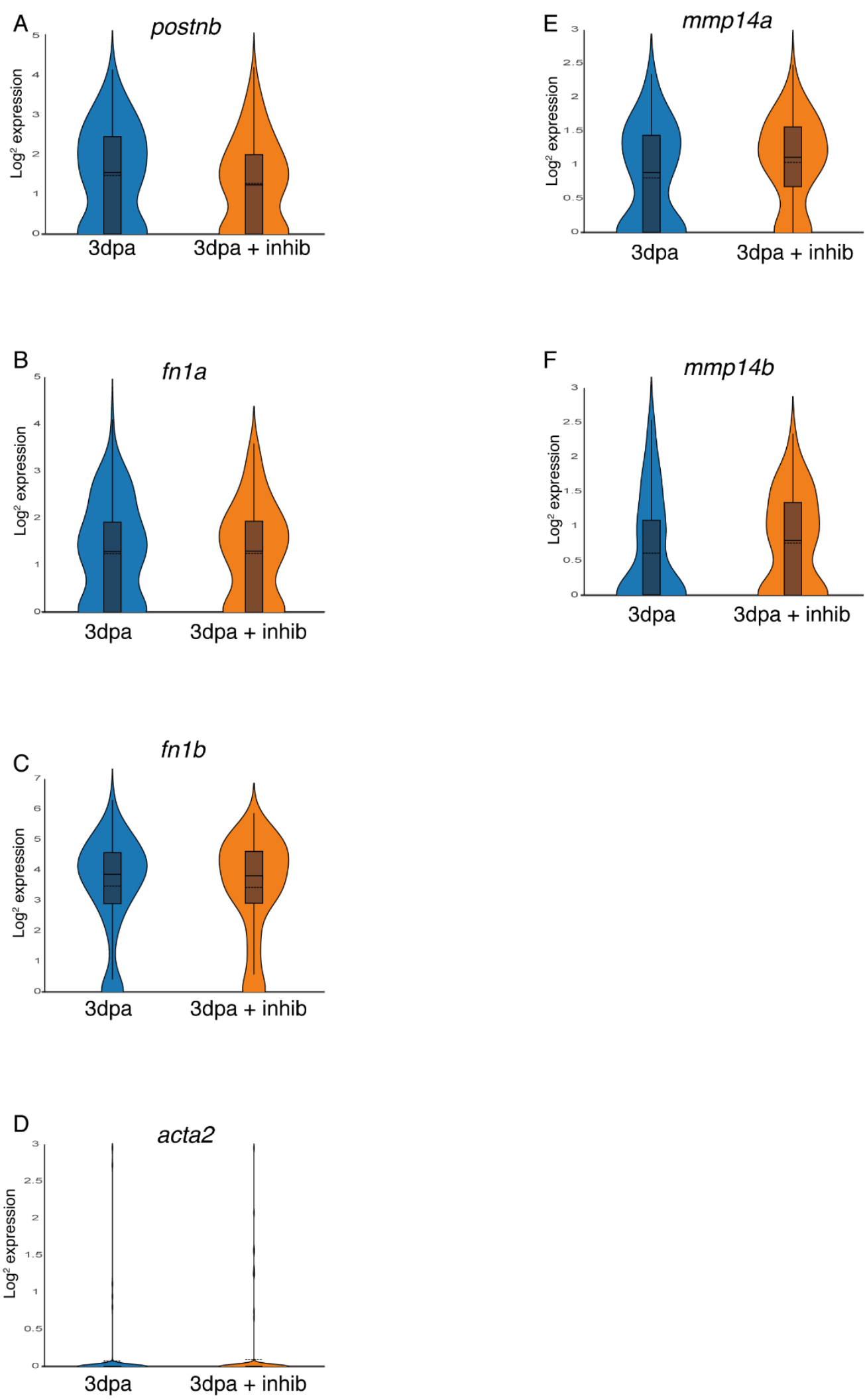
bioRxiv preprint doi: https://doi.org/10.1101/2021.10.25.465720; this version posted October 26, 2021. The copyright holder for this preprint (which was not certified by peer review) is the author/funder. All rights reserved. No reuse allowed without permission.

1050 Supplemental Figure 11. Comparison of gene expression by fibroblasts following Mmp14 inhibition.

1051 (A-F) Violin plots comparing the expression of different genes within the fibroblast population in untreated

1052 (ctrl) or MMP14 inhibitor treated (+inhib) 3dpa regenerating zebrafish hearts, postnb (A), fnla (B), fnlb

1053 (C), $\operatorname{acta2}(\mathbf{D})$, mmpl4a (E) and mmpl4b (F). 

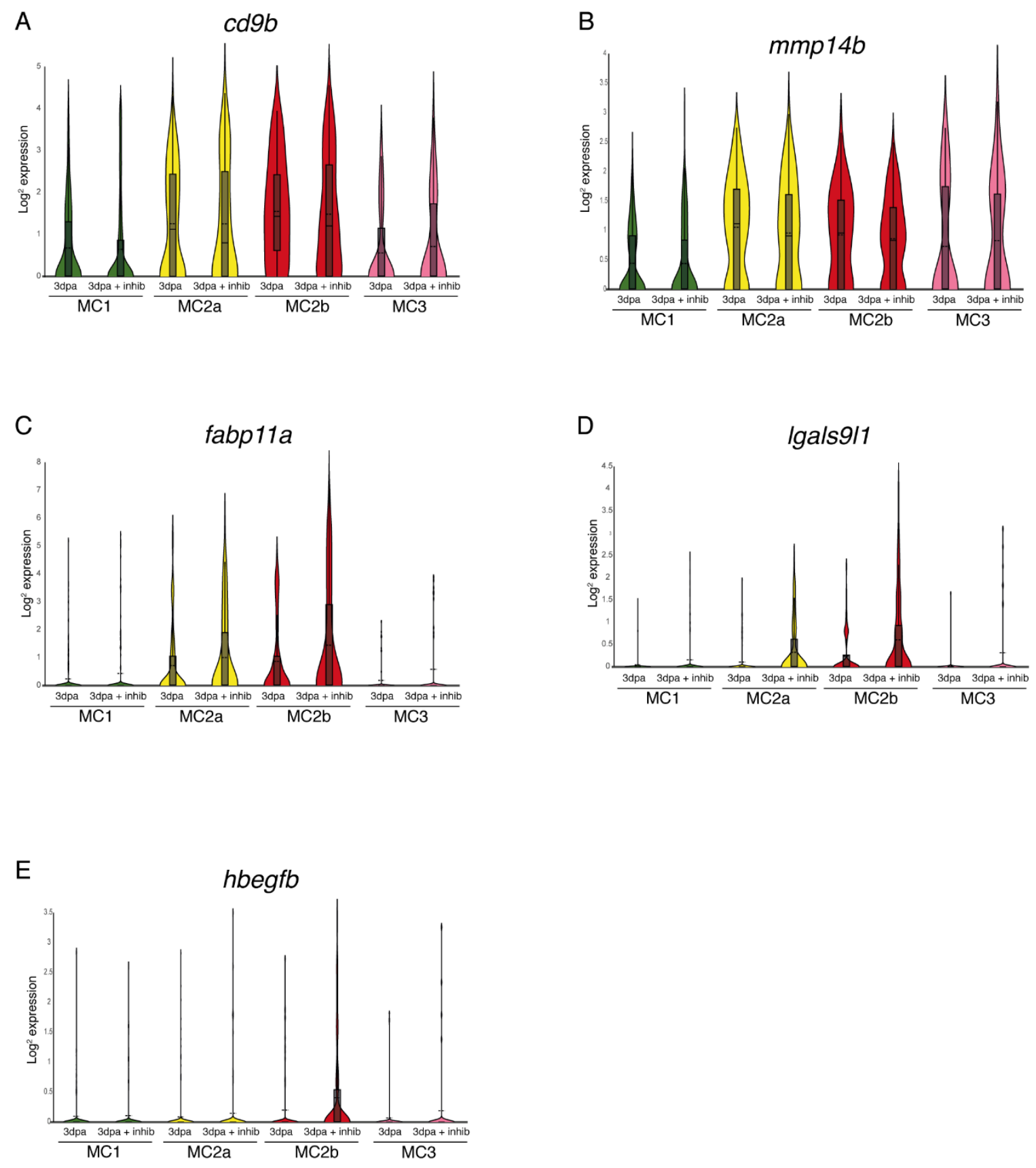
1056 Supplemental Figure 12. Comparison of gene expression within the macrophage subpopulations

1057 following Mmp14 inhibition. (A-E) Violin plots comparing the expression of different genes within the 1058 macrophage subpopulations (MC1, MC2a, MC2b and MC3) in untreated (ctrl) or MMP14 inhibitor treated 1059 (+inhib) 3dpa regenerating zebrafish hearts, cd9b (A), mmp14b (B), fabpl 1a (C), lgals9l1 (D), and hbegfb 1060 (E).

1061 